\title{
To which world regions does the valence- dominance model of social perception apply?
}

\begin{abstract}
Over the past 10 years, Oosterhof and Todorov's valence-dominance model has emerged as the most prominent account of how people evaluate faces on social dimensions. In this model, two dimensions (valence and dominance) underpin social judgements of faces. Because this model has primarily been developed and tested in Western regions, it is unclear whether these findings apply to other regions. We addressed this question by replicating Oosterhof and Todorov's methodology across 11 world regions, 41 countries and 11,570 participants. When we used Oosterhof and Todorov's original analysis strategy, the valence-dominance model generalized across regions. When we used an alternative methodology to allow for correlated dimensions, we observed much less generalization. Collectively, these results suggest that, while the valence-dominance model generalizes very well across regions when dimensions are forced to be orthogonal, regional differences are revealed when we use different extraction methods and correlate and rotate the dimension reduction solution.
\end{abstract}

\section{Protocol registration}

The stage 1 protocol for this Registered Report was accepted in principle on 5 November 2018. The protocol, as accepted by the journal, can be found at https://doi.org/10.6084/m9.figshare.7611443.v1.

$P$ eople quickly and involuntarily form impressions of others based on their facial appearance ${ }^{1-3}$. These impressions then influence important social outcomes ${ }^{4,5}$. For example, people are more likely to cooperate in socioeconomic interactions with individuals whose faces are evaluated as more trustworthy ${ }^{6}$, vote for individuals whose faces are evaluated as more competent ${ }^{7}$, and seek romantic relationships with individuals whose faces are evaluated as more attractive ${ }^{8}$. Facial appearance can even influence life-or-death outcomes. For example, untrustworthy-looking defendants are more likely to receive death sentences?. Given that such evaluations influence profound outcomes, understanding how people evaluate others' faces can provide insight into a potentially important route through which social stereotypes impact behaviour ${ }^{10,11}$.

Over the past decade, Oosterhof and Todorov's valence-dominance model $^{12}$ has emerged as the most prominent account of how we evaluate faces on social dimensions ${ }^{5}$. Oosterhof and Todorov identified 13 different traits (aggressiveness, attractiveness, caringness, confidence, dominance, emotional stability, unhappiness, intelligence, meanness, responsibility, sociability, trustworthiness and weirdness) that perceivers spontaneously use to evaluate faces when forming trait impressions ${ }^{12}$. From these traits, they derived a two-dimensional model of perception: valence and dominance. Valence, best characterized by rated trustworthiness, was defined as the extent to which the target was perceived as having the intention to harm the viewer ${ }^{12}$. Dominance, best characterized by rated dominance, was defined as the extent to which the target was perceived as having the ability to inflict harm on the viewer ${ }^{12}$. Crucially, the model proposes that these two dimensions are sufficient to drive social evaluations of faces. As a consequence, the majority of research on the effects of social evaluations of faces has focused on one or both of these dimensions $s^{4,5}$.

Successful replications of the valence-dominance model have only been conducted in Western samples ${ }^{13,14}$. This focus on the West is consistent with research on human behaviour more broadly, which typically draws general assumptions from analyses of Western participants' responses ${ }^{15}$. Kline et al. ${ }^{16}$ recently termed this problematic practice the Western centrality assumption and argued that regional variation, rather than universality, is probably the default for human behaviour.

Consistent with Kline et al's notion that human behaviour is best characterized by regional variation, two recent studies of social evaluation of faces by Chinese participants indicate that different factors underlie their impressions ${ }^{17,18}$. Both studies reported that Chinese participants' social evaluations of faces were underpinned by a valence dimension similar to that reported by Oosterhof and Todorov for Western participants, but not by a corresponding dominance dimension. Instead, both studies reported a second dimension, referred to as capability, which was best characterized by rated intelligence. Furthermore, the ethnicity of the faces rated only subtly affected perceptions ${ }^{17}$. Research into potential cultural differences in the effects of experimentally manipulated facial characteristics on social perceptions has also found little evidence that cultural differences in social perceptions of faces depend on the ethnicity of the faces presented ${ }^{19-21}$. Collectively, these results suggest that the Western centrality assumption may be an important barrier to understanding how people evaluate faces on social dimensions. Crucially, these studies also suggest that the valence-dominance model is not necessarily a universal account of social evaluations of faces and warrants further investigation in the broadest set of samples possible.

Although the studies described above demonstrate that the valence-dominance model is not perfectly universal, to which specific world regions it does and does not apply are open and important questions. Demonstrating differences between British and Chinese raters is evidence against the universality of the valencedominance model, but it does not adequately address these questions. Social perception in China may be unique in not fitting the valence-dominance model because of the atypically high general importance placed on status-related traits, such as capability, during social interactions in China ${ }^{22,23}$. Indeed, Tan et al..$^{24}$ demonstrated face-processing differences between Chinese participants living in mainland China and Chinese participants living in nearby countries, such as Malaysia. Insights regarding the unique formation of social perceptions in other cultures and world regions are lacking. 
Only a large-scale study investigating social perceptions in many different world regions can provide such insights.

To establish the world regions to which the valence-dominance model applies, we replicated Oosterhof and Todorov's methodology $^{12}$ in a wide range of world regions (Africa, Asia, Australia and New Zealand, Central America and Mexico, Eastern Europe, the Middle East, the United States and Canada, Scandinavia, South America, the United Kingdom and Western Europe; see Table 1). Our study is the most comprehensive test of social evaluations of faces to date, including more than 11,000 participants. Participating research groups were recruited via the Psychological Science Accelerator project ${ }^{25-27}$. Previous studies compared two cultures to demonstrate regional differences ${ }^{17,18}$. In contrast, the scale and scope of our study allows us to generate the most comprehensive picture of the world regions to which the valence-dominance model does and does not apply.

We tested two specific competing predictions: (1) the valencedominance model applies to all world regions; and (2) the valencedominance model applies in Western-world regions, but not other world regions.

\section{Results}

Analysed dataset. Following the planned data exclusions (see the Supplementary Information for a breakdown of these exclusions; code 1.5), the analysed dataset is summarized in Table 2.

Main analysis (principal component analysis (PCA); code 2.1). Oosterhof and Todorov reported the results of a PCA with orthogonal components, no rotation and retaining components with eigenvalues of $>1$. We conducted an identical analysis and report: (1) the number of components extracted per the registered criteria; (2) whether the first and second components had the same primary pattern as Oosterhof and Todorov reported; and (3) the similarity of the first and second factors as quantified with a congruence coefficient.

We extracted the same number of components (two) as Oosterhof and Todorov in two world regions (Africa and South America) and a different number of components (three) in the other world regions (see Fig. 1). In the world regions where a third component was extracted, the trait ratings of unhappy and weird tended to have the highest loadings on that component, but those ratings also crossloaded on the first component. We hesitate to interpret or describe this component with any authority because it varied across world regions, consisted of crossloaded traits and explained only a small proportion of additional variance.

The primary pattern reported by Oosterhof and Todorov (a first component that correlated strongly with rated trustworthiness but not with rated dominance and a second component that correlated strongly with rated dominance but not with rated trustworthiness) was present in all world regions except Eastern Europe. In Eastern Europe, dominance was correlated with the first component more strongly than our registered criterion (i.e., that dominance would correlate weakly with the first component; $r<0.5$ ). Figure 1 shows the full loading matrices for each region and Table 3 shows how these relate to our registered criteria.

We report Tucker's coefficient of congruence, $\phi$, which quantifies the loading similarity of Oosterhof and Todorov's reported component to the corresponding component we extracted. However, it is important to interpret $\phi$ with caution when the numbers of components differ across the solutions being compared. When comparing loadings across solutions, an assumption is that the configuration of the traits to components is the same (that is, configural invariance). To the extent that the structures of the loading matrices differ across solutions, the comparability of the loadings is compromised (that is, loadings estimated from different dimensional spaces are not on the same scale). For world regions that did not have the same configuration of traits to components (that is, those with a different number
Table 1 | World regions, countries and localities of data collection

\begin{tabular}{|c|c|}
\hline World region & Countries and localities \\
\hline Africa & Kenya, (Nigeria) and South Africa \\
\hline Asia & China, India, Malaysia, Taiwan and Thailand \\
\hline $\begin{array}{l}\text { Australia and New } \\
\text { Zealand }\end{array}$ & Australia and New Zealand \\
\hline $\begin{array}{l}\text { Central America and } \\
\text { Mexico }\end{array}$ & El Salvador and Mexico \\
\hline Eastern Europe & $\begin{array}{l}\text { Hungary, Lithuania, Poland, Russia, Serbia and } \\
\text { Slovakia }\end{array}$ \\
\hline The Middle East & Iran, Israel and Turkey \\
\hline $\begin{array}{l}\text { United States and } \\
\text { Canada }\end{array}$ & Canada and the United States \\
\hline Scandinavia & Denmark, (Finland), Norway and (Sweden) \\
\hline South America & $\begin{array}{l}\text { Argentina, Brazil, Chile, Colombia and } \\
\text { Ecuador }\end{array}$ \\
\hline United Kingdom & England, Scotland and Wales \\
\hline Western Europe & $\begin{array}{l}\text { Austria, Belgium, France, Germany, (Greece), } \\
\text { Italy, the Netherlands, Portugal, Spain and } \\
\text { Switzerland }\end{array}$ \\
\hline \multicolumn{2}{|c|}{$\begin{array}{l}\text { We collected data from a minimum of } 350 \text { raters per world region based on the simulations } \\
\text { described in the Methods. Countries in parentheses were added to the list after acceptance in } \\
\text { principle of the stage } 1 \text { protocol. Ecuador was incorrectly classified as Central America and Mexico } \\
\text { in our stage } 1 \text { submission, but has been classified as South America for analyses and in our stage } \\
2 \text { submission. }\end{array}$} \\
\hline
\end{tabular}

of components extracted or a different primary pattern observed), $\phi$ was uninterpretable. This is because the differences in configuration across the two solutions were conflated with the loading differences.

Our analyses indicated that the first component was equal to the first component in Oosterhof and Todorov's original study for all world regions $(\phi>0.95)$. The second component was equal to $(\phi>0.95)$ or fairly similar to $(\phi>0.85)$ the second component reported by Oosterhof and Todorov in all of the world regions except Asia $(\phi=0.848)$. Table 4 summarizes these results.

Together, these results suggest that the valence-dominance model generalizes across world regions when using an identical analysis to that used in Oosterhof and Todorov's original study. Thus, the results of our PCA support prediction 1 (that the valencedominance model will apply to all world regions) but not prediction 2 (that the valence-dominance model will apply in Western-world regions but not other world regions). However, we note here that in most world regions we extracted a third component not extracted in the original study: that Eastern Europe did not demonstrate the same primary pattern and that $\phi$ should be interpreted with caution for all world regions except Africa and South America.

Robustness analyses (exploratory factor analysis (EFA); code 2.2). Following our analysis plan, we conducted additional robustness analyses that directly addressed criticisms of the type of statistical analyses used by Oosterhof and Todorov (see ref. ${ }^{28}$ for a discussion of these criticisms). These robustness analyses employed EFA with an oblimin rotation as the model and used parallel analysis to identify the number of factors to extract. The goal of an EFA with an oblimin rotation is to simplify the loading matrix and yield interpretable factors.

We conducted this analysis on Oosterhof and Todorov's original data and found a similar result to their PCA solution: two factors extracted, with factor 1 characterized by a high loading for trustworthiness and factor 2 characterized by a high loading for dominance. 
Table 2 | Number of participants per region and Cronbach's $\alpha$ values following data quality checks and exclusions

\begin{tabular}{|c|c|c|c|c|c|c|c|c|c|c|c|c|c|}
\hline Region & Aggressive & Attractive & Caring & Confident & Dominant & $\begin{array}{l}\text { Emotionally } \\
\text { stable }\end{array}$ & Intelligent & Mean & Responsible & Sociable & Trustworthy & Unhappy & Weird \\
\hline Western Europe & $\begin{array}{l}\alpha=0.978 \\
n=152\end{array}$ & $\begin{array}{l}\alpha=0.991 \\
n=147\end{array}$ & $\begin{array}{l}\alpha=0.976 \\
n=136\end{array}$ & $\begin{array}{l}\alpha=0.985 \\
n=156\end{array}$ & $\begin{array}{l}\alpha=0.973 \\
n=150\end{array}$ & $\begin{array}{l}\alpha=0.981 \\
n=141\end{array}$ & $\begin{array}{l}\alpha=0.975 \\
n=141\end{array}$ & $\begin{array}{l}\alpha=0.969 \\
n=120\end{array}$ & $\begin{array}{l}\alpha=0.978 \\
n=138\end{array}$ & $\begin{array}{l}\alpha=0.988 \\
n=188\end{array}$ & $\begin{array}{l}\alpha=0.978 \\
n=141\end{array}$ & $\begin{array}{l}\alpha=0.983 \\
n=140\end{array}$ & $\begin{array}{l}\alpha=0.982 \\
n=113\end{array}$ \\
\hline $\begin{array}{l}\text { United States and } \\
\text { Canada }\end{array}$ & $\begin{array}{l}\alpha=0.983 \\
n=248\end{array}$ & $\begin{array}{l}\alpha=0.991 \\
n=224\end{array}$ & $\begin{array}{l}\alpha=0.986 \\
n=257\end{array}$ & $\begin{array}{l}\alpha=0.989 \\
n=303\end{array}$ & $\begin{array}{l}\alpha=0.977 \\
n=246\end{array}$ & $\begin{array}{l}\alpha=0.986 \\
n=270\end{array}$ & $\begin{array}{l}\alpha=0.979 \\
n=239\end{array}$ & $\begin{array}{l}\alpha=0.984 \\
n=270\end{array}$ & $\begin{array}{l}\alpha=0.984 \\
n=269\end{array}$ & $\begin{array}{l}\alpha=0.988 \\
n=246\end{array}$ & $\begin{array}{l}\alpha=0.984 \\
n=263\end{array}$ & $\begin{array}{l}\alpha=0.985 \\
n=252\end{array}$ & $\begin{array}{l}\alpha=0.987 \\
n=226\end{array}$ \\
\hline United Kingdom & $\begin{array}{l}\alpha=0.879 \\
n=16\end{array}$ & $\begin{array}{l}\alpha=0.949 \\
n=22\end{array}$ & $\begin{array}{l}\alpha=0.936 \\
n=34\end{array}$ & $\begin{array}{l}\alpha=0.93 \\
n=30\end{array}$ & $\begin{array}{l}\alpha=0.886 \\
n=34\end{array}$ & $\begin{array}{l}\alpha=0.9 \\
n=30\end{array}$ & $\begin{array}{l}\alpha=0.911 \\
n=34\end{array}$ & $\begin{array}{l}\alpha=0.87 \\
n=27\end{array}$ & $\begin{array}{l}\alpha=0.892 \\
n=37\end{array}$ & $\begin{array}{l}\alpha=0.932 \\
n=28\end{array}$ & $\begin{array}{l}\alpha=0.92 \\
n=27\end{array}$ & $\begin{array}{l}\alpha=0.937 \\
n=24\end{array}$ & $\begin{array}{l}\alpha=0.899 \\
n=18\end{array}$ \\
\hline South America & $\begin{array}{l}\alpha=0.948 \\
n=97\end{array}$ & $\begin{array}{l}\alpha=0.982 \\
n=108\end{array}$ & $\begin{array}{l}\alpha=0.944 \\
n=112\end{array}$ & $\begin{array}{l}\alpha=0.968 \\
n=108\end{array}$ & $\begin{array}{l}\alpha=0.957 \\
n=121\end{array}$ & $\begin{array}{l}\alpha=0.949 \\
n=100\end{array}$ & $\begin{array}{l}\alpha=0.938 \\
n=110\end{array}$ & $\begin{array}{l}\alpha=0.949 \\
n=95\end{array}$ & $\begin{array}{l}\alpha=0.937 \\
n=117\end{array}$ & $\begin{array}{l}\alpha=0.974 \\
n=110\end{array}$ & $\begin{array}{l}\alpha=0.952 \\
n=107\end{array}$ & $\begin{array}{l}\alpha=0.961 \\
n=87\end{array}$ & $\begin{array}{l}\alpha=0.973 \\
n=116\end{array}$ \\
\hline Scandinavia & $\begin{array}{l}\alpha=0.95 \\
n=48\end{array}$ & $\begin{array}{l}\alpha=0.969 \\
n=44\end{array}$ & $\begin{array}{l}\alpha=0.949 \\
n=46\end{array}$ & $\begin{array}{l}\alpha=0.96 \\
n=56\end{array}$ & $\begin{array}{l}\alpha=0.941 \\
n=49\end{array}$ & $\begin{array}{l}\alpha=0.955 \\
n=67\end{array}$ & $\begin{array}{l}\alpha=0.958 \\
n=54\end{array}$ & $\begin{array}{l}\alpha=0.912 \\
n=36\end{array}$ & $\begin{array}{l}\alpha=0.915 \\
n=37\end{array}$ & $\begin{array}{l}\alpha=0.969 \\
n=64\end{array}$ & $\begin{array}{l}\alpha=0.949 \\
n=58\end{array}$ & $\begin{array}{l}\alpha=0.952 \\
n=55\end{array}$ & $\begin{array}{l}\alpha=0.952 \\
n=39\end{array}$ \\
\hline Middle East & $\begin{array}{l}\alpha=0.912 \\
n=32\end{array}$ & $\begin{array}{l}\alpha=0.949 \\
n=32\end{array}$ & $\begin{array}{l}\alpha=0.934 \\
n=42\end{array}$ & $\begin{array}{l}\alpha=0.943 \\
n=39\end{array}$ & $\begin{array}{l}\alpha=0.9 \\
n=35\end{array}$ & $\begin{array}{l}\alpha=0.903 \\
n=33\end{array}$ & $\begin{array}{l}\alpha=0.896 \\
n=48\end{array}$ & $\begin{array}{l}\alpha=0.901 \\
n=36\end{array}$ & $\begin{array}{l}\alpha=0.87 \\
n=34\end{array}$ & $\begin{array}{l}\alpha=0.944 \\
n=41\end{array}$ & $\begin{array}{l}\alpha=0.895 \\
n=42\end{array}$ & $\begin{array}{l}\alpha=0.943 \\
n=57\end{array}$ & $\begin{array}{l}\alpha=0.896 \\
n=32\end{array}$ \\
\hline Eastern Europe & $\begin{array}{l}\alpha=0.941 \\
n=59\end{array}$ & $\begin{array}{l}\alpha=0.971 \\
n=58\end{array}$ & $\begin{array}{l}\alpha=0.926 \\
n=56\end{array}$ & $\begin{array}{l}\alpha=0.946 \\
n=60\end{array}$ & $\begin{array}{l}\alpha=0.952 \\
n=74\end{array}$ & $\begin{array}{l}\alpha=0.923 \\
n=56\end{array}$ & $\begin{array}{l}\alpha=0.939 \\
n=64\end{array}$ & $\begin{array}{l}\alpha=0.937 \\
n=68\end{array}$ & $\begin{array}{l}\alpha=0.953 \\
n=65\end{array}$ & $\begin{array}{l}\alpha=0.955 \\
n=68\end{array}$ & $\begin{array}{l}\alpha=0.937 \\
n=54\end{array}$ & $\begin{array}{l}\alpha=0.964 \\
n=74\end{array}$ & $\begin{array}{l}\alpha=0.956 \\
n=53\end{array}$ \\
\hline $\begin{array}{l}\text { Central America } \\
\text { and Mexico }\end{array}$ & $\begin{array}{l}\alpha=0.845 \\
n=26\end{array}$ & $\begin{array}{l}\alpha=0.93 \\
n=25\end{array}$ & $\begin{array}{l}\alpha=0.788 \\
n=24\end{array}$ & $\begin{array}{l}\alpha=0.89 \\
n=32\end{array}$ & $\begin{array}{l}\alpha=0.859 \\
n=33\end{array}$ & $\begin{array}{l}\alpha=0.835 \\
n=23\end{array}$ & $\begin{array}{l}\alpha=0.832 \\
n=33\end{array}$ & $\begin{array}{l}\alpha=0.817 \\
n=23\end{array}$ & $\begin{array}{l}\alpha=0.824 \\
n=22\end{array}$ & $\begin{array}{l}\alpha=0.882 \\
n=28\end{array}$ & $\begin{array}{l}\alpha=0.851 \\
n=27\end{array}$ & $\begin{array}{l}\alpha=0.771 \\
n=27\end{array}$ & $\begin{array}{l}\alpha=0.842 \\
n=15\end{array}$ \\
\hline $\begin{array}{l}\text { Australia and } \\
\text { New Zealand }\end{array}$ & $\begin{array}{l}\alpha=0.956 \\
n=77\end{array}$ & $\begin{array}{l}\alpha=0.98 \\
n=88\end{array}$ & $\begin{array}{l}\alpha=0.964 \\
n=90\end{array}$ & $\begin{array}{l}\alpha=0.972 \\
n=93\end{array}$ & $\begin{array}{l}\alpha=0.936 \\
n=66\end{array}$ & $\begin{array}{l}\alpha=0.957 \\
n=88\end{array}$ & $\begin{array}{l}\alpha=0.951 \\
n=81\end{array}$ & $\begin{array}{l}\alpha=0.947 \\
n=71\end{array}$ & $\begin{array}{l}\alpha=0.937 \\
n=68\end{array}$ & $\begin{array}{l}\alpha=0.972 \\
n=95\end{array}$ & $\begin{array}{l}\alpha=0.953 \\
n=72\end{array}$ & $\begin{array}{l}\alpha=0.948 \\
n=85\end{array}$ & $\begin{array}{l}\alpha=0.962 \\
n=70\end{array}$ \\
\hline Asia & $\begin{array}{l}\alpha=0.932 \\
n=59\end{array}$ & $\begin{array}{l}\alpha=0.957 \\
n=52\end{array}$ & $\begin{array}{l}\alpha=0.948 \\
n=73\end{array}$ & $\begin{array}{l}\alpha=0.959 \\
n=72\end{array}$ & $\begin{array}{l}\alpha=0.917 \\
n=55\end{array}$ & $\begin{array}{l}\alpha=0.908 \\
n=55\end{array}$ & $\begin{array}{l}\alpha=0.927 \\
n=64\end{array}$ & $\begin{array}{l}\alpha=0.909 \\
n=51\end{array}$ & $\begin{array}{l}\alpha=0.931 \\
n=63\end{array}$ & $\begin{array}{l}\alpha=0.952 \\
n=65\end{array}$ & $\begin{array}{l}\alpha=0.93 \\
n=61\end{array}$ & $\begin{array}{l}\alpha=0.937 \\
n=61\end{array}$ & $\begin{array}{l}\alpha=0.942 \\
n=49\end{array}$ \\
\hline Africa & $\begin{array}{l}\alpha=0.808 \\
n=45\end{array}$ & $\begin{array}{l}\alpha=0.873 \\
n=38\end{array}$ & $\begin{array}{l}\alpha=0.865 \\
n=44\end{array}$ & $\begin{array}{l}\alpha=0.805 \\
n=31\end{array}$ & $\begin{array}{l}\alpha=0.79 \\
n=38\end{array}$ & $\begin{array}{l}\alpha=0.779 \\
n=38\end{array}$ & $\begin{array}{l}\alpha=0.756 \\
n=37\end{array}$ & $\begin{array}{l}\alpha=0.889 \\
n=51\end{array}$ & $\begin{array}{l}\alpha=0.811 \\
n=36\end{array}$ & $\begin{array}{l}\alpha=0.819 \\
n=34\end{array}$ & $\begin{array}{l}\alpha=0.867 \\
n=49\end{array}$ & $\begin{array}{l}\alpha=0.795 \\
n=43\end{array}$ & $\begin{array}{l}\alpha=0.889 \\
n=37\end{array}$ \\
\hline
\end{tabular}

However, for all other world regions, we extracted more than two factors using parallel analysis. Full EFA loading matrices for each region and Oosterhof and Todorov's original data are shown in Fig. 2. The four-factor solution for the USA and Canada did not converge. We did not register a contingency for nonconvergence, but because parallel analysis can lead to over extraction, we reran the EFA with one fewer than the number of suggested factors. The model converged when estimating three factors.

In contrast with the PCA, the results of our robustness analyses showed less evidence that the valence-dominance model generalizes across world regions. For example, we extracted a different number of factors than the original solution for all world regions. A summary of the results for our replication criteria is given in Table 5 .

Because the number of factors differed from the original solution in all world regions and the loading matrices were differentially rotated from the original solution, it is not valid to compare the differences in the loadings from the original solution with those observed in the world regions reported here, as we had initially planned. Loadings quantify the relationship of traits to a factor. To compare loadings across samples, we must first determine whether we extracted the same factor in each sample (that is, satisfied the assumption of configural invariance). Our registered analyses included the calculation of Tucker's coefficient of congruence, $\phi$ in order to compare the first factor from the original study with the first factor we extracted in a given world region, and to compare the second factor from the original study with the second factor extracted in a given world region. However, because we extracted a different number of factors from the original solution in all world regions, it is not valid to compare the loadings across these different factors, or to quantify their differences using $\phi$.

The congruence coefficient is only appropriate to report when we can ensure that the factors are comparable across samples. That the number of factors extracted did not replicate the original pattern and that the EFAs were rotated differently across world regions negates the comparability of the loadings. Consistent with our registered analysis code, we reported $\phi$ for the relationship of the first factor from Oosterhof and Todorov to the factor with the most explained variance in a world region, and $\phi$ for the relationship of the second factor from Oosterhof and Todorov to the factor with the second most explained variance in a world region only in the Supplementary Information. However, we stress that these coefficients are quantifying loadings that link to different factors from different dimensional spaces and are not necessarily comparable.

In summary, the results of our EFA support neither prediction 1 (that the valence-dominance model will apply to all world regions) nor prediction 2 (that the valence-dominance model will apply to Western-world regions but not other world regions).

\section{Discussion}

Our primary analyses-PCAs identical to those reported by Oosterhof and Todorov-suggested that the valence-dominance model of social perception of faces generalizes well across world regions. Although most world regions showed a third component not discussed in the original work ${ }^{12}$, this third component is actually similar to the third component in Oosterhof and Todorov's original study. In Oosterhof and Todorov's original study, they did not interpret the third component because its eigenvalue was below 1 , whereas in our analyses the eigenvalues of the third components in most of the regions were just above 1 . Nonetheless, the third component in each region had a factor congruence between 0.77 and 0.90 with the third component for Oosterhof and Todorov's data. However, we emphasize here that many of these dimensions accounted for a relatively small proportion of the variance explained and, thus, may be of limited theoretical importance.

In contrast with the results of our PCAs, an alternative analysis that addressed common criticisms of the type of analysis Oosterhof and Todorov employed showed much less generalization across world regions. We used modern extraction techniques and EFAs with correlated factor rotations. The correlated rotation methods aim to simplify the loading matrix with the goal of estimating 
Component 1 Component 2 Component 3

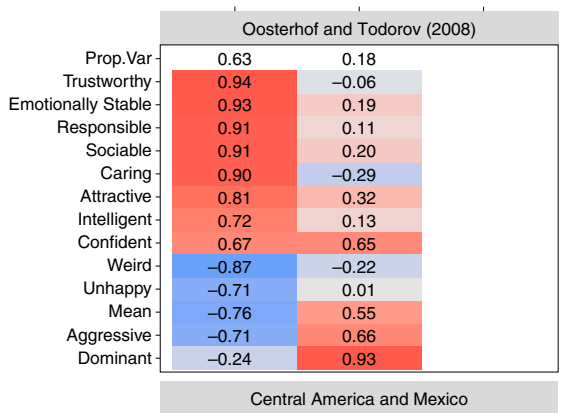

Central America and Mexico

\begin{tabular}{|c|c|c|c|}
\hline \multirow{3}{*}{$\begin{array}{r}\text { Prop.Var } \\
\text { Trustworthy }\end{array}$} & \\
\hline & 0.56 & 0.18 & 0.08 \\
\hline & 0.92 & -0.05 & -0.14 \\
\hline Emotionally Stable & 0.92 & 0.06 & 0.10 \\
\hline Responsible & 0.85 & 0.36 & -0.03 \\
\hline Sociable - & 0.84 & -0.14 & 0.33 \\
\hline Caring - & 0.78 & -0.41 & 0.07 \\
\hline Attractive - & 0.67 & 0.46 & -0.30 \\
\hline Intelligent & 0.76 & 0.27 & -0.28 \\
\hline Confident - & 0.74 & 0.45 & 0.36 \\
\hline Weird- & -0.71 & -0.15 & 0.50 \\
\hline Unhappy & -0.74 & 0.07 & -0.58 \\
\hline Mean- & -0.75 & 0.48 & 0.14 \\
\hline Aggressive - & -0.62 & 0.69 & 0.16 \\
\hline Dominant - & 0.01 & 0.92 & 0.07 \\
\hline
\end{tabular}

\begin{tabular}{|c|c|c|c|}
\hline \multirow{3}{*}{$\begin{array}{r}\text { Prop.Var } \\
\text { Trustworthy }\end{array}$} & \multicolumn{3}{|c|}{ South America } \\
\hline & 0.59 & 0.21 & 0.08 \\
\hline & 0.90 & -0.15 & -0.24 \\
\hline Emotionally Stable & 0.95 & 0.02 & 0.09 \\
\hline Responsible - & 0.82 & 0.18 & -0.21 \\
\hline Sociable & 0.90 & -0.05 & 0.37 \\
\hline Caring & 0.76 & -0.55 & 0.12 \\
\hline Attractive & 0.71 & 0.48 & -0.21 \\
\hline Intelligent & 0.77 & 0.24 & -0.32 \\
\hline Confident & 0.79 & 0.50 & 0.31 \\
\hline Weird & -0.77 & -0.22 & 0.42 \\
\hline Unhappy & -0.79 & 0.01 & -0.57 \\
\hline Mean - & -0.73 & 0.64 & 0.09 \\
\hline Aggressive & -0.60 & 0.76 & 0.09 \\
\hline Dominant & 0.31 & 0.91 & 0.08 \\
\hline
\end{tabular}

\begin{tabular}{|rr|}
\hline & \multicolumn{1}{|c|}{ Africa } \\
\hline 0.57 & 0.19 \\
0.92 & -0.07 \\
0.82 & 0.13 \\
0.78 & 0.34 \\
0.89 & -0.05 \\
0.81 & -0.41 \\
0.78 & 0.34 \\
0.75 & 0.24 \\
0.69 & 0.53 \\
-0.82 & -0.10 \\
-0.75 & 0.25 \\
-0.70 & 0.63 \\
-0.58 & 0.75 \\
0.27 & 0.84 \\
\hline
\end{tabular}

\begin{tabular}{|rrr|}
\hline \multicolumn{3}{|c|}{ Asia } \\
\hline 0.64 & 0.18 & 0.09 \\
0.92 & -0.01 & -0.27 \\
0.88 & -0.14 & 0.22 \\
0.91 & 0.25 & -0.05 \\
0.93 & -0.24 & 0.16 \\
0.91 & -0.30 & 0.09 \\
0.76 & 0.51 & -0.24 \\
0.83 & 0.42 & -0.08 \\
0.81 & 0.26 & 0.47 \\
-0.76 & -0.27 & 0.52 \\
-0.73 & 0.36 & -0.53 \\
-0.80 & 0.50 & 0.15 \\
-0.65 & 0.63 & 0.33 \\
0.37 & 0.86 & 0.22 \\
\hline
\end{tabular}

\begin{tabular}{|rrr|}
\multicolumn{3}{|c|}{ Eastern Europe } \\
\hline 0.65 & 0.16 & 0.09 \\
0.94 & -0.11 & 0.20 \\
0.90 & 0.05 & -0.28 \\
0.92 & 0.19 & 0.14 \\
0.90 & -0.11 & -0.25 \\
0.87 & -0.28 & 0.11 \\
0.88 & 0.23 & 0.24 \\
0.87 & 0.08 & 0.21 \\
0.77 & 0.53 & -0.30 \\
-0.81 & -0.12 & -0.41 \\
-0.60 & 0.11 & 0.76 \\
-0.64 & 0.72 & -0.02 \\
-0.63 & 0.74 & -0.02 \\
0.60 & 0.76 & -0.01 \\
\hline
\end{tabular}

\begin{tabular}{|c|c|c|}
\hline \multicolumn{3}{|c|}{ United Kingdom } \\
\hline 0.60 & 0.18 & 0.10 \\
\hline 0.94 & -0.12 & -0.10 \\
\hline 0.89 & 0.06 & 0.18 \\
\hline 0.87 & 0.24 & -0.23 \\
\hline 0.92 & -0.11 & 0.25 \\
\hline 0.87 & -0.31 & 0.12 \\
\hline 0.73 & 0.48 & -0.31 \\
\hline 0.80 & 0.15 & -0.32 \\
\hline 0.78 & 0.41 & 0.39 \\
\hline-0.76 & -0.23 & 0.46 \\
\hline-0.65 & 0.24 & -0.66 \\
\hline-0.67 & 0.63 & 0.12 \\
\hline-0.62 & 0.71 & 0.18 \\
\hline 0.33 & 0.85 & 0.23 \\
\hline
\end{tabular}

Component 1 Component 2 Component 3

\begin{tabular}{|rrr|}
\hline \multicolumn{3}{|c|}{ Middle East } \\
\hline 0.54 & 0.25 & 0.09 \\
0.83 & -0.38 & -0.21 \\
0.94 & -0.06 & 0.04 \\
0.77 & 0.39 & 0.11 \\
0.87 & -0.28 & 0.28 \\
0.83 & -0.41 & 0.06 \\
0.66 & 0.48 & -0.38 \\
0.72 & 0.52 & -0.19 \\
0.81 & 0.45 & 0.26 \\
-0.70 & -0.10 & 0.61 \\
-0.72 & 0.10 & -0.58 \\
-0.44 & 0.84 & 0.09 \\
-0.55 & 0.77 & 0.13 \\
0.49 & 0.81 & 0.11 \\
\hline
\end{tabular}

Component 1 Component 2 Component 3

\begin{tabular}{|rrr|}
\hline \multicolumn{4}{|c|}{ Australia and New Zealand } \\
\hline 0.63 & 0.17 & 0.10 \\
0.94 & -0.08 & -0.19 \\
0.92 & 0.10 & 0.22 \\
0.91 & 0.16 & -0.21 \\
0.93 & -0.10 & 0.24 \\
0.90 & -0.25 & 0.17 \\
0.75 & 0.44 & -0.30 \\
0.79 & 0.14 & -0.27 \\
0.79 & 0.45 & 0.36 \\
-0.77 & -0.13 & 0.52 \\
-0.68 & 0.13 & -0.69 \\
-0.67 & 0.65 & 0.07 \\
-0.74 & 0.62 & 0.13 \\
0.26 & 0.91 & 0.11 \\
\hline & Scandinavia & \\
\hline 0.64 & 0.18 & 0.08 \\
0.95 & -0.12 & -0.14 \\
0.95 & 0.04 & 0.13 \\
0.91 & 0.12 & -0.12 \\
0.94 & -0.11 & 0.20 \\
0.88 & -0.30 & 0.08 \\
0.80 & 0.35 & -0.31 \\
0.79 & 0.25 & -0.26 \\
0.77 & 0.50 & 0.34 \\
-0.75 & -0.15 & 0.52 \\
-0.74 & 0.07 & -0.62 \\
-0.69 & 0.68 & 0.06 \\
-0.63 & 0.72 & 0.03 \\
0.39 & 0.88 & 0.11 \\
\hline & & \\
\hline & & \\
\hline
\end{tabular}

\begin{tabular}{|c|c|c|}
\hline \multicolumn{3}{|c|}{ United States and Canada } \\
\hline 0.66 & 0.17 & 0.08 \\
\hline 0.97 & -0.07 & -0.17 \\
\hline 0.95 & 0.01 & 0.16 \\
\hline 0.92 & 0.13 & -0.23 \\
\hline 0.95 & -0.04 & 0.21 \\
\hline 0.89 & -0.30 & 0.14 \\
\hline 0.72 & 0.48 & -0.27 \\
\hline 0.83 & 0.17 & -0.27 \\
\hline 0.81 & 0.42 & 0.37 \\
\hline-0.79 & -0.20 & 0.45 \\
\hline-0.73 & 0.19 & -0.62 \\
\hline-0.70 & 0.68 & 0.05 \\
\hline-0.70 & 0.67 & 0.18 \\
\hline 0.41 & 0.84 & 0.16 \\
\hline
\end{tabular}

\begin{tabular}{|rrr|}
\hline \multicolumn{3}{|c|}{ Western Europe } \\
\hline 0.65 & 0.19 & 0.08 \\
0.96 & -0.17 & 0.14 \\
0.95 & 0.08 & -0.17 \\
0.90 & 0.30 & 0.11 \\
0.93 & -0.19 & -0.22 \\
0.86 & -0.36 & 0.04 \\
0.82 & 0.36 & 0.31 \\
0.81 & 0.24 & 0.24 \\
0.83 & 0.45 & -0.30 \\
-0.78 & -0.15 & -0.50 \\
-0.74 & 0.06 & 0.61 \\
-0.64 & 0.73 & -0.05 \\
-0.69 & 0.70 & 0.01 \\
0.36 & 0.88 & -0.15 \\
\hline
\end{tabular}

Fig. 1 | PCA loading matrices for each region. Positive loadings are shaded red and negative loadings are shaded blue. Darker colours correspond to stronger loadings. The proportion of variance (Prop.Var) explained by each component is included at the top of each table.

Table 3 | Replication criteria for the PCA for each region

\begin{tabular}{|c|c|c|c|c|c|}
\hline \multirow[t]{2}{*}{ Region } & \multicolumn{2}{|l|}{ Component 1} & \multicolumn{2}{|c|}{ Component 2} & \multirow[t]{2}{*}{ Replicated } \\
\hline & Trustworthy & Dominant & Dominant & Trustworthy & \\
\hline Oosterhof and Todorov ${ }^{12}$ & 0.941 & -0.244 & 0.929 & -0.060 & Yes \\
\hline Africa & 0.924 & 0.271 & 0.843 & -0.065 & Yes \\
\hline Asia & 0.922 & 0.370 & 0.863 & -0.006 & Yes \\
\hline Australia and New Zealand & 0.943 & 0.257 & 0.907 & -0.076 & Yes \\
\hline Central America and Mexico & 0.918 & 0.007 & 0.915 & -0.050 & Yes \\
\hline Eastern Europe & 0.938 & 0.599 & 0.755 & -0.113 & No \\
\hline Middle East & 0.831 & 0.490 & 0.810 & -0.382 & Yes \\
\hline Scandinavia & 0.953 & 0.392 & 0.881 & -0.121 & Yes \\
\hline South America & 0.898 & 0.309 & 0.905 & -0.151 & Yes \\
\hline United Kingdom & 0.944 & 0.331 & 0.851 & -0.121 & Yes \\
\hline United States and Canada & 0.966 & 0.406 & 0.841 & -0.073 & Yes \\
\hline Western Europe & 0.957 & 0.357 & 0.875 & -0.166 & Yes \\
\hline
\end{tabular}

Oosterhof and Todorov's valence-dominance model was judged to have been replicated in a given world region if the first component had a loading of $>0.7$ with trustworthiness and $<0.5$ with dominance, and if the second component had a loading of $>0.7$ with dominance and $<0.5$ with trustworthiness.

interpretable factors, and in our data revealed more regional variation. These results suggest that, if the dimensions of face perception are indeed correlated, using analytical techniques that force these dimensions to be uncorrelated may be obscuring important regional differences in the structure of face perceptions.
A necessary next step for moving forward in person perception research is to address which analysis model (PCA or EFA) best aligns with theory, so that models and theories can be revised and expanded appropriately in future research. Crucially, the two models make different assumptions about trait ratings of faces. 
Table 4 | Factor congruence for each region's PCA

\begin{tabular}{|c|c|c|c|c|}
\hline \multirow[t]{2}{*}{ Region } & \multicolumn{2}{|c|}{ Component 1} & \multicolumn{2}{|c|}{ Component 2} \\
\hline & Loading & Congruence & Loading & Congruence \\
\hline Africa & 0.980 & Equal & 0.947 & Fairly similar \\
\hline Asia & 0.974 & Equal & 0.843 & Not similar \\
\hline $\begin{array}{l}\text { Australia and } \\
\text { New Zealand }\end{array}$ & 0.982 & Equal & 0.959 & Equal \\
\hline $\begin{array}{l}\text { Central America } \\
\text { and Mexico }\end{array}$ & 0.992 & Equal & 0.935 & Fairly similar \\
\hline Eastern Europe & 0.953 & Equal & 0.948 & Fairly similar \\
\hline Middle East & 0.952 & Equal & 0.859 & Fairly similar \\
\hline Scandinavia & 0.973 & Equal & 0.960 & Equal \\
\hline South America & 0.976 & Equal & 0.953 & Equal \\
\hline United Kingdom & 0.976 & Equal & 0.938 & Fairly similar \\
\hline $\begin{array}{l}\text { United States and } \\
\text { Canada }\end{array}$ & 0.972 & Equal & 0.952 & Equal \\
\hline Western Europe & 0.975 & Equal & 0.936 & Fairly similar \\
\hline
\end{tabular}

The PCA model does not assume that a latent factor causes the trait ratings of the faces. The component captures linear combinations of the original variables, maximized to explain variance. Furthermore, in the original valence-dominance model, those components were assumed to be orthogonal. In contrast, the theory underlying the EFA model is that a latent factor causes the trait ratings and any unexplained variance in that rating is measurement error. Additionally, our EFA models allowed for the factors to be correlated.

Theory can guide which model we use to analyse person perception data. A person perception theory that aligns with a PCA model would state that there are no underlying latent factors that cause a person to rate a face in a particular way. There are, instead, perceptual processes that vary across contexts, those doing the rating and those being rated, and the differential processes give rise to components that can be used to reduce the data. This theory of person perception would move forward with identifying the shared processes across contexts, raters and ratees to see whether there are predictable patterns in how the data are reduced.

A person perception theory that aligns with an EFA model makes different assumptions about the processes that give rise to face ratings. This theory would state that latent factors (for example, valence or dominance) cause the trait ratings and, once we account for the correct latent factors, any variability left in the ratings is measurement error. We suggest that more careful and explicit consideration of how theory connects to these approaches, and of which approach is best suited to different research questions, will benefit the field.

Our study is one of several recent studies that have begun to utilize different statistical models and to explore more dynamic theories of trait ratings ${ }^{21,29,30}$ by exploring how the structures of trait ratings vary systematically. This growing body of work catalogues variations in trait ratings by target demographic ${ }^{21,29,31}$, target status $^{32}$, target age ${ }^{33}$, perceiver knowledge ${ }^{34}$ and cultural factors ${ }^{17,18}$. Furthermore, this growing body of work proposes dynamic theories of person perception and more flexible statistical models for capturing them ${ }^{21,29,30,35}$.

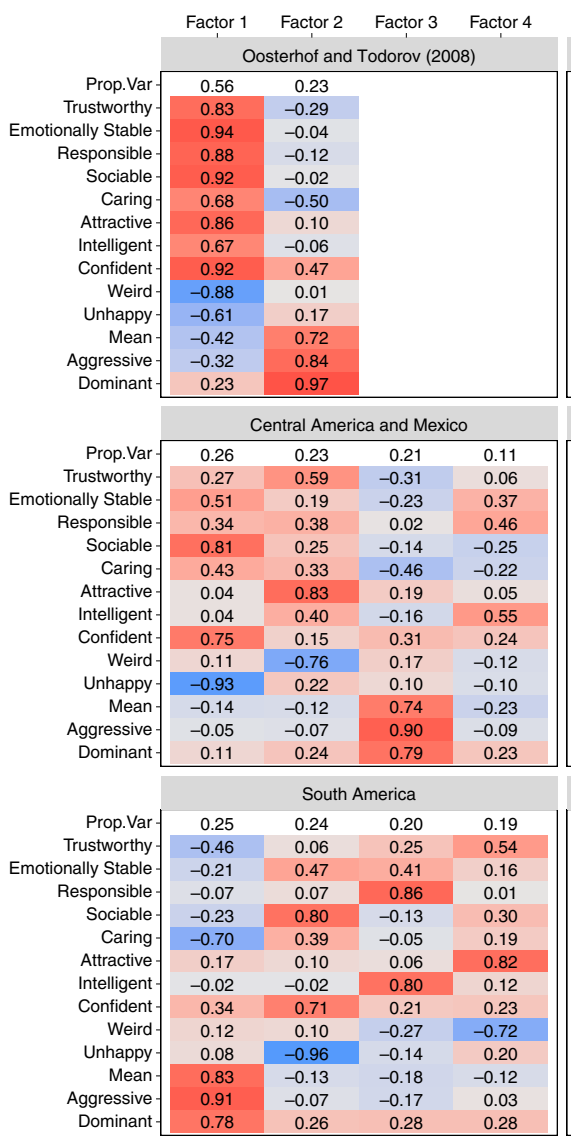

\begin{tabular}{|c|c|c|c|}
\hline Factor 1 & Factor 2 & Factor 3 & Factor 4 \\
\hline \multicolumn{4}{|c|}{ Africa } \\
\hline 0.37 & 0.25 & 0.16 & \\
\hline 0.79 & 0.21 & -0.13 & \\
\hline 0.28 & 0.62 & 0.27 & \\
\hline 0.76 & 0.05 & 0.21 & \\
\hline 0.68 & 0.28 & -0.08 & \\
\hline 0.34 & 0.57 & -0.28 & \\
\hline 0.96 & -0.16 & 0.12 & \\
\hline 0.59 & 0.18 & 0.17 & \\
\hline 0.31 & 0.45 & 0.63 & \\
\hline-0.94 & 0.09 & 0.09 & \\
\hline 0.07 & -0.94 & -0.06 & \\
\hline-0.17 & -0.65 & 0.48 & \\
\hline-0.24 & -0.42 & 0.68 & \\
\hline 0.20 & 0.07 & 0.80 & \\
\hline \multicolumn{4}{|c|}{ Eastern Europe } \\
\hline 0.49 & 0.19 & 0.19 & \\
\hline 0.84 & -0.32 & 0.03 & \\
\hline 0.50 & -0.09 & 0.54 & \\
\hline 0.89 & -0.02 & 0.09 & \\
\hline 0.47 & -0.25 & 0.51 & \\
\hline 0.64 & -0.43 & 0.12 & \\
\hline 0.95 & 0.02 & -0.03 & \\
\hline 0.83 & -0.12 & 0.03 & \\
\hline 0.57 & 0.41 & 0.54 & \\
\hline-0.92 & 0.09 & 0.17 & \\
\hline 0.17 & 0.12 & -1.01 & \\
\hline-0.23 & 0.82 & -0.16 & \\
\hline-0.21 & 0.85 & -0.16 & \\
\hline 0.75 & 0.61 & 0.15 & \\
\hline \multicolumn{4}{|c|}{ United Kingdom } \\
\hline 0.26 & 0.25 & 0.18 & 0.18 \\
\hline 0.34 & 0.51 & -0.30 & 0.19 \\
\hline 0.47 & 0.03 & -0.09 & 0.58 \\
\hline 0.06 & 0.45 & -0.06 & 0.54 \\
\hline 0.73 & 0.32 & -0.13 & 0.04 \\
\hline 0.64 & 0.48 & -0.32 & -0.18 \\
\hline-0.01 & 0.82 & 0.22 & 0.16 \\
\hline-0.08 & 0.31 & -0.19 & 0.68 \\
\hline 0.67 & 0.13 & 0.39 & 0.34 \\
\hline 0.09 & -0.81 & 0.06 & -0.17 \\
\hline-0.95 & 0.23 & 0.07 & -0.06 \\
\hline-0.23 & -0.08 & 0.72 & -0.21 \\
\hline-0.15 & -0.08 & 0.85 & -0.18 \\
\hline 0.25 & 0.27 & 0.77 & 0.21 \\
\hline
\end{tabular}

\begin{tabular}{|c|c|c|c|}
\hline Factor 1 & Factor 2 & Factor 3 & Factor 4 \\
\hline \multicolumn{4}{|c|}{ Asia } \\
\hline 0.37 & 0.36 & 0.16 & \\
\hline 0.76 & 0.24 & -0.25 & \\
\hline 0.26 & 0.72 & -0.09 & \\
\hline 0.69 & 0.36 & 0.08 & \\
\hline 0.26 & 0.75 & -0.20 & \\
\hline 0.28 & 0.69 & -0.29 & \\
\hline 0.90 & 0.01 & 0.20 & \\
\hline 0.77 & 0.23 & 0.21 & \\
\hline 0.18 & 0.83 & 0.41 & \\
\hline-1.01 & 0.17 & 0.14 & \\
\hline 0.25 & -1.06 & 0.06 & \\
\hline-0.31 & -0.46 & 0.57 & \\
\hline-0.31 & -0.26 & 0.74 & \\
\hline 0.49 & 0.11 & 0.79 & \\
\hline \multicolumn{4}{|c|}{ Middle East } \\
\hline 0.29 & 0.28 & 0.26 & \\
\hline 0.18 & -0.69 & 0.44 & \\
\hline 0.54 & -0.36 & 0.38 & \\
\hline 0.56 & 0.10 & 0.41 & \\
\hline 0.74 & -0.47 & -0.01 & \\
\hline 0.45 & -0.63 & 0.16 & \\
\hline 0.07 & 0.10 & 0.82 & \\
\hline 0.27 & 0.16 & 0.71 & \\
\hline 0.80 & 0.21 & 0.29 & \\
\hline 0.18 & 0.30 & -0.89 & \\
\hline-0.89 & 0.18 & 0.21 & \\
\hline-0.03 & 0.93 & 0.06 & \\
\hline-0.06 & 0.92 & -0.06 & \\
\hline 0.50 & 0.60 & 0.45 & \\
\hline \multicolumn{4}{|c|}{ United States and Canada } \\
\hline 0.41 & 0.28 & 0.19 & \\
\hline 0.77 & 0.19 & -0.30 & \\
\hline 0.47 & 0.57 & -0.12 & \\
\hline 0.87 & 0.11 & -0.11 & \\
\hline 0.39 & 0.64 & -0.14 & \\
\hline 0.31 & 0.54 & -0.41 & \\
\hline 0.86 & -0.00 & 0.22 & \\
\hline 0.80 & 0.07 & -0.07 & \\
\hline 0.35 & 0.76 & 0.38 & \\
\hline-0.97 & 0.15 & 0.08 & \\
\hline 0.22 & -1.05 & 0.13 & \\
\hline-0.19 & -0.27 & 0.79 & \\
\hline-0.32 & -0.12 & 0.84 & \\
\hline 0.49 & 0.26 & 0.72 & \\
\hline
\end{tabular}

\begin{tabular}{|c|c|c|c|}
\hline Factor 1 & Factor 2 & Factor 3 & Factor 4 \\
\hline \multicolumn{4}{|c|}{ Australia and New Zealand } \\
\hline 0.30 & 0.23 & 0.22 & 0.15 \\
\hline 0.73 & 0.28 & 0.06 & -0.08 \\
\hline 0.17 & 0.43 & 0.57 & 0.05 \\
\hline 0.54 & 0.44 & 0.06 & 0.11 \\
\hline 0.64 & -0.01 & 0.50 & -0.03 \\
\hline 0.74 & -0.07 & 0.39 & -0.16 \\
\hline 0.63 & 0.31 & -0.12 & 0.39 \\
\hline-0.09 & 0.94 & 0.13 & -0.06 \\
\hline 0.12 & 0.28 & 0.66 & 0.45 \\
\hline-0.42 & -0.64 & 0.22 & -0.02 \\
\hline-0.00 & 0.03 & -0.98 & 0.07 \\
\hline-0.31 & -0.25 & -0.19 & 0.64 \\
\hline-0.50 & -0.21 & -0.11 & 0.63 \\
\hline 0.16 & 0.07 & 0.12 & 0.87 \\
\hline \multicolumn{4}{|c|}{ Scandinavia } \\
\hline 0.41 & 0.27 & 0.20 & \\
\hline 0.74 & 0.21 & -0.30 & \\
\hline 0.50 & 0.54 & -0.10 & \\
\hline 0.75 & 0.23 & -0.06 & \\
\hline 0.39 & 0.61 & -0.23 & \\
\hline 0.42 & 0.43 & -0.42 & \\
\hline 0.91 & -0.01 & 0.15 & \\
\hline 0.78 & 0.07 & 0.05 & \\
\hline 0.32 & 0.72 & 0.41 & \\
\hline-0.98 & 0.22 & 0.06 & \\
\hline 0.18 & -1.04 & 0.10 & \\
\hline-0.31 & -0.20 & 0.79 & \\
\hline-0.22 & -0.21 & 0.80 & \\
\hline 0.43 & 0.29 & 0.81 & \\
\hline \multicolumn{4}{|c|}{ Western Europe } \\
\hline 0.27 & 0.24 & 0.24 & 0.17 \\
\hline 0.40 & 0.16 & 0.56 & -0.17 \\
\hline 0.34 & 0.56 & 0.24 & 0.03 \\
\hline 0.58 & 0.22 & 0.29 & 0.19 \\
\hline 0.03 & 0.57 & 0.51 & -0.10 \\
\hline-0.00 & 0.21 & 0.80 & -0.21 \\
\hline 0.55 & -0.06 & 0.49 & 0.30 \\
\hline 1.03 & 0.11 & -0.18 & -0.08 \\
\hline 0.26 & 0.68 & 0.11 & 0.42 \\
\hline-0.68 & 0.20 & -0.42 & -0.03 \\
\hline 0.02 & -0.99 & 0.06 & 0.06 \\
\hline-0.13 & -0.18 & -0.38 & 0.70 \\
\hline-0.17 & -0.29 & -0.31 & 0.71 \\
\hline 0.11 & 0.26 & 0.14 & 0.87 \\
\hline
\end{tabular}

Fig. 2 | EFA loading matrices for each region. Positive loadings are shaded red and negative loadings are shaded blue. Darker colours correspond to stronger loadings. The proportion of variance explained by each factor is included at the top of each table. 
Table 5 | Replication criteria for the EFA for each region

Region

Oosterhof and Todorov

Africa

Asia

Australia and New Zealand

Central America and Mexico

Eastern Europe

Middle East

Scandinavia

South America

United Kingdom

United States and Canada

Western Europe

Factor 1

Factor 2

Trustworthy

0.826

0.786

0.761

0.730

0.268

0.843

0.177

0.744

$-0.458$

0.338

0.768

0.398
Dominant

0.970

0.069

0.110

0.071

0.241

0.609

0.600

0.293

0.261

0.778

0.249

0.491

0.111

\section{NATURE HUMAN BEHAVIOUR}

Oosterhof and Todorov's valence-dominance model was judged to have been replicated in a given world region if the first factor had a loading $>0.7$ with trustworthiness and $<0.5$ with dominance and the second factor had a loading $>0.7$ with dominance and $<0.5$ with trustworthiness.

Our results are consistent with this recent work in that they do not provide strong evidence that there are a few generalizable latent factors that cause the trait ratings across world regions. However, they do suggest a dynamic process of person perception and elucidate the differential patterns of ratings across world regions. We can use these data, representing impressions formed on a global scale, to expand or refine our theories and to guide the selection of statistical models to represent those theories. Given the accumulating evidence for variation in trait ratings, it is important that the connection between the statistical models used to represent theories of person perception are explicit and can accommodate the complexities of the impression formation process.

\section{Methods}

Ethics. Each research group had approval from their local ethics committee or institutional review board to conduct the study, had explicitly indicated that their institution did not require approval for the researchers to conduct this type of face-rating task or had explicitly indicated that the current study was covered by a pre-existing approval. Although the specifics of the consent procedure differed across research groups, all participants provided informed consent. All data were stored centrally on University of Glasgow servers.

Procedure. Oosterhof and Todorov derived their valence-dominance model from a PCA of ratings (by US raters) of 66 faces for 13 different traits (aggressiveness, attractiveness, caringness, confidence, dominance, emotional stability, intelligence, meanness, responsibility, sociability, trustworthiness, unhappiness and weirdness $)^{12}$. Using the criteria of the number of components with eigenvalues greater than 1.0, this analysis produced two principal components. The first component explained $63 \%$ of the variance in trait ratings, strongly correlated with rated trustworthiness $(r=0.94)$ and weakly correlated with rated dominance $(r=-0.24)$. The second component explained $18 \%$ of the variance in trait ratings, strongly correlated with rated dominance $(r=0.93)$ and weakly correlated with rated trustworthiness $(r=-0.06)$. We replicated Oosterhof and Todorov's method ${ }^{12}$ and primary analysis in each world region we examined.

Stimuli in our study came from an open-access, full-colour face image set ${ }^{36}$ consisting of images of the faces of 60 men and 60 women taken under standardized photographic conditions $\left(M_{\text {age }}=26.4\right.$ years; s.d. $=3.6$ years; range $=18-35$ years $)$. These 120 images consisted of 30 Black ( 15 male; 15 female), 30 White ( 15 male; 15 female), 30 Asian (15 male; 15 female) and 30 Latin faces (15 male; 15 female). As reported by Oosterhof and Todorov's study ${ }^{12}$, the individuals photographed posed looking directly at the camera with a neutral expression, and the background, lighting and clothing (here, a grey t-shirt) were constant across images.

In our study, adult raters were randomly assigned to rate the 13 adjectives tested by Oosterhof and Todorov using scales ranging from 1 (not at all) to 9 (very) for all 120 faces in a fully randomized order at their own pace. Because all researchers collected data through an identical interface (except for differences in instruction language), data collection protocols were highly standardized across laboratories. Each participant completed the block of 120 face-rating trials twice so that we could report test-retest reliabilities of ratings; ratings from the first and second blocks were averaged for all analyses (see code 1.5.5 in the Supplementary Information).

Raters also completed a short questionnaire requesting demographic information (sex, age and ethnicity). These variables were not considered in Oosterhof and Todorov's analyses but were collected in our study so that other researchers could use them in secondary analyses of the published data. The data from this study comprise the largest and most comprehensive open-access set of face ratings with open stimuli from around the world, providing an invaluable resource for further research addressing the Western centrality assumption in person perception research.

Raters completed the task in a language appropriate for their country (see below). To mitigate potential problems with translating single-word labels, dictionary definitions for each of the 13 traits were provided. Twelve of these dictionary definitions had previously been used to test for effects of social impressions on the memorability of face photographs ${ }^{37}$. Dominance (not included in that study) was defined as strong and important.

Participants. Simulations determined that we should obtain at least 25 different raters for each of the 13 traits in every region (see https://osf.io/x7fus/ for code and data). We focused on ratings of attractiveness and intelligence for the simulations because they showed the highest and lowest agreement among the traits analysed by Oosterhof and Todorov, respectively. First, we sampled from a population of 2,513 raters, each of whom had rated the attractiveness of 102 faces; these simulations showed that more than $99 \%$ of 1,000 random samples of 25 raters produced good or excellent inter-rater reliability coefficients (Cronbach's $\alpha$ values $>0.80$ ). We then repeated these simulations, sampling from a population of 37 raters, each of whom rated the intelligence of 100 faces, showing that $93 \%$ of 1,000 random samples of 25 raters produced good or excellent inter-rater reliability coefficients (Cronbach's $\alpha$ values $>0.80$ ). Thus, averages of ratings from 25 or more raters will have produced reliable dependent variables in our analyses; we planned to test at least 9,000 raters in total.

In addition to rating the faces for the 13 traits examined by Oosterhof and Todorov, 25 participants in each region were randomly assigned to rate the targets' age in light of Sutherland et al's results showing that a youth/attractiveness dimension emerged from analyses of a sample of faces with a very diverse age range $^{38}$. Age ratings were not included in analyses relating to replications of Oosterhof and Todorov's valence-dominance model. These age ratings were collected to allow for planned exploratory analyses including rated age, but we did not perform these analyses.

Analysis plan. The code used for our analyses is included in the Supplementary Information and publicly available from the Open Science Framework (https://osf. io/87rbg/). The specific sections of code are cited below.

Ratings from each world region were analysed separately and anonymous raw data have been published on the Open Science Framework. Our main analyses directly replicated the PCA reported by Oosterhof and Todorov to test their theoretical model in each region sampled (code 2.1). First, we calculated the average rating for each face separately for each of the 13 traits (code 2.1.2). We then subjected 
these mean ratings to PCA with orthogonal components and no rotation, as Oosterhof and Todorov did (code 2.1.3). Using the criteria they reported, we retained and interpreted components with eigenvalues greater than 1.0 (code 2.1.3.1).

Criteria for replicating Oosterhof and Todorov's valence-dominance model. We used multiple sources of evidence to judge whether Oosterhof and Todorov's valence-dominance model replicated in a given world region. First, we examined the solution from the PCA conducted in each region and determined whether Oosterhof and Todorov's primary pattern replicated according to three criteria: (1) the first two components had eigenvalues greater than 1.0 ; (2) the first component (that is, the one explaining more of the variance in ratings) correlated strongly with trustworthiness $(r>0.7)$ and weakly with dominance $(r<0.5)$; and (3) the second component (that is, the one explaining less of the variance in ratings) correlated strongly with dominance $(r>0.7)$ and weakly with trustworthiness $(r<0.5)$. If the solution in a world region met all three of these criteria, we concluded that the primary pattern of the model replicated in that region (code 2.1.3.3).

In addition to reporting whether the primary pattern was replicated in each region, we also reported Tucker's coefficient of congruence ${ }^{39,40}$. The congruence coefficient, $\phi$, ranges from -1 to 1 and quantifies the similarity between two vectors of loadings ${ }^{41}$. It is:

$$
\phi(x, y)=\frac{\sum x_{i} y_{i}}{\sqrt{\sum x_{i}^{2} \sum y_{i}^{2}}}
$$

where $x_{i}$ and $y_{i}$ are the loadings of variable $i(i=1, \ldots, n$ number of indicators in the analysis) onto factors $x$ and $y$, respectively. For the purposes of the current research, we compared the vector of loadings from the first component from Oosterhof and Todorov with the vector of loadings from the first component estimated from each world region. We repeated this analysis for the second component. This produced a standardized measure of component similarity for each component in each world region that was not sensitive to the mean size of the loadings ${ }^{42}$. Furthermore, this coefficient was fitting for the current study because it does not require an a priori specification of a factor structure for each group, as would be needed if we were to compare the factor structures in a multiple-group confirmatory factor analysis. Following previous guidelines ${ }^{42}$, we concluded that the components reported by Oosterhof and Todorov were not similar to those estimated in a given world region if the coefficient was $<0.85$, were fairly similar if it was between 0.85 and 0.94 and were equal if it was $>0.95$ (code 2.1.4).

Thus, we reported whether the solution had the same primary pattern that Oosterhof and Todorov found and quantified the degree of similarity between each component and the corresponding component from Oosterhof and Todorov's work. This connects to our competing predictions.

Prediction 1 (the valence-dominance model applies to all world regions) was supported if the solution from the PCA conducted in each region satisfied all of the criteria described above. Specifically, the primary pattern was replicated and the components had at least a fair degree of similarity as quantified by a value of $\phi$ of 0.85 or greater.

Prediction 2 (the valence-dominance model applies in Western-world regions but not other world regions) was supported if the solutions from the PCA conducted in Australia and New Zealand, the United States and Canada, Scandinavia, the United Kingdom and Western Europe, but not Africa, Asia, Central America and Mexico, Eastern Europe, the Middle East or South America, satisfied the criteria described above.

Exclusions. Data from raters who failed to complete all 120 ratings in the first block of trials or who provided the same rating for $75 \%$ or more of the faces were excluded from the analyses (codes 1.5.1, 1.5.3 and 1.5.5).

Data quality checks. Following previous research testing the valence-dominance model ${ }^{12-14}$, data quality was checked by separately calculating the inter-rater agreement (indicated by Cronbach's $\alpha$ and test-retest reliability) for each trait in every world region (code 2.1.1). A trait was only included in the analysis for that region if the coefficient exceeded 0.70. Cases in which the coefficient did not exceed 0.70 are reported and discussed. There were no cases in which the coefficient did not exceed 0.70. Test-retest reliability of traits was not used to exclude traits from analysis.

Power analysis. Simulations showed that we had more than $95 \%$ power to detect the key effect of interest (that is, two components meeting the criteria for replicating Oosterhof and Todorov's work, as described above). We used the open data from Morrison et al.s replication ${ }^{13}$ of Oosterhof and Todorov's research to generate a variance-covariance matrix representative of typical inter-relationships among the 13 traits tested in our study. We then generated 1,000 samples of 120 faces from these distributions and ran our planned PCA (which is identical to that reported by Oosterhof and Todorov) on each sample (see https://osf.io/87rbg/ for code and data). The results of $>99 \%$ of these analyses matched our criteria for replicating Oosterhof and Todorov's findings. Thus, 120 faces gave us more than $95 \%$ power to replicate Oosterhof and Todorov's results.

Robustness analyses. Oosterhof and Todorov extracted and interpreted components with an eigenvalue greater than 1.0 using an unrotated PCA. As described above, we directly replicated their method in our main analyses but acknowledge that this type of analysis has been criticized.

First, it has been argued that EFA with rotation, rather than an unrotated PCA, is more appropriate when one intends to measure correlated latent factors, as was the case in the current study ${ }^{43,44}$. Second, the extraction rule of eigenvalues greater than 1.0 has been criticized for not indicating the optimal number of components, as well as for producing unreliable components ${ }^{45,46}$.

To address these limitations, we repeated our main analyses using EFA with an oblimin rotation as the model and a parallel analysis to determine the number of factors to extract. We also recalculated the congruence coefficient described above for these EFA results (code 2.2.2).

We used parallel analysis to determine the number of factors to extract because it has been described as yielding the optimal number of components (or factors) across the largest array of scenarios ${ }^{43,47,48}$ (code 2.2.1). In a parallel analysis, random data matrices are generated such that they have the same number of cases and variables as the real data. The mean eigenvalue from the components of the random data is compared with the eigenvalue for each component from the real data. Components are then retained if their eigenvalues exceed those from the randomly generated data ${ }^{49}$.

The purpose of these additional analyses was twofold: (1) to address potential methodological limitations in the original study; and (2) to ensure that the results of our replication of Oosterhof and Todorov's study are robust to the implementation of those more rigorous analytical techniques. The same criteria for replicating Oosterhof and Todorov's model described above were applied to this analysis (code 2.2.1.3).

Reporting Summary. Further information on research design is available in the Nature Research Reporting Summary linked to this article.

\section{Data availability}

Full data are publicly available at https://osf.io/87rbg/.

\section{Code availability}

Full analysis code is publicly available at https://osf.io/87rbg/.

Received: 18 May 2018; Accepted: 23 October 2020;

Published online: 4 January 2021

\section{References}

1. Olivola, C. Y. \& Todorov, A. Elected in 100 milliseconds: appearance-based trait inferences and voting. J. Nonverbal Behav. 34, 83-110 (2010).

2. Ritchie, K. L., Palermo, R. \& Rhodes, G. Forming impressions of facial attractiveness is mandatory. Sci. Rep. 7, 469 (2017).

3. Willis, J. \& Todorov, A. First impressions: making up your mind after $100 \mathrm{~ms}$ exposure to a face. Psychol. Sci. 17, 592-598 (2006).

4. Olivola, C. Y., Funk, F. \& Todorov, A. Social attributions from faces bias human choices. Trends Cogn. Sci. 18, 566-570 (2014).

5. Todorov, A., Olivola, C. Y., Dotsch, R. \& Mende-Siedlecki, P. Social attributions from faces: determinants, consequences, accuracy, and functional significance. Annu. Rev. Psychol. 66, 519-545 (2015).

6. Van 't Wout, M. \& Sanfey, A. G. Friend or foe: the effect of implicit trustworthiness judgments in social decision-making. Cognition 108, 796-803 (2008).

7. Todorov, A., Mandisodza, A. N., Goren, A. \& Hall, C. C. Inferences of competence from faces predict election outcomes. Science 308, 1623-1626 (2005).

8. Langlois, J. H. et al. Maxims or myths of beauty? A meta-analytic and theoretical review. Psychol. Bull. 126, 390-423 (2000).

9. Wilson, J. P. \& Rule, N. O. Facial trustworthiness predicts extreme criminal-sentencing outcomes. Psychol. Sci. 26, 1325-1331 (2015).

10. Todorov, A., Said, C. P., Engell, A. D. \& Oosterhof, N. N. Understanding evaluation of faces on social dimensions. Trends Cogn. Sci. 12, 455-460 (2008).

11. Jack, R. E. \& Schyns, P. G. Toward a social psychophysics of face communication. Annu. Rev. Psychol. 68, 269-297 (2017).

12. Oosterhof, N. N. \& Todorov, A. The functional basis of face evaluation. Proc. Natl Acad. Sci. USA 105, 11087-11092 (2008).

13. Morrison, D., Wang, H., Hahn, A. C., Jones, B. C. \& DeBruine, L. M. Predicting the reward value of faces and bodies from social perception. PLOS ONE 12, e0185093 (2017).

14. Wang, H., Hahn, A. C., DeBruine, L. M. \& Jones, B. C. The motivational salience of faces is related to both their valence and dominance. PLoS ONE 11, e0161114 (2016).

15. Henrich, J., Heine, S. \& Norenzayan, A. The weirdest people in the world? Behav. Brain Sci. 33, 61-83 (2010).

16. Kline, M. A., Shamsudheen, R. \& Broesch, T. Variation is the universal: making cultural evolution work in developmental psychology. Phil. Trans. $R$. Soc. B 373, 20170059 (2018). 
17. Sutherland, C. A. M. et al. Facial first impressions across culture: data-driven modeling of Chinese and British perceivers' unconstrained facial impressions. Pers. Soc. Psychol. Bull. 44, 521-537 (2018).

18. Wang, H. et al. A data-driven study of Chinese participants' social judgments of Chinese faces. PLoS ONE 14, e0210315 (2019).

19. Han, C. et al. Cultural differences in preferences for facial coloration. Evol. Hum. Behav. 39, 154-159 (2018).

20. Perrett, D. I. et al. Effects of sexual dimorphism on facial attractiveness. Nature 394, 884-887 (1998).

21. Xie, S. Y., Flake, J. K. \& Hehman, E.Perceiver and target characteristics contribute to impression formation differently across race and gender. J. Pers. Soc. Psychol. 117, 364-385 (2019).

22. Li, N. P., Valentine, K. A. \& Patel, L. Mate preferences in the US and Singapore: a cross-cultural test of the mate preference priority model. Pers. Individ. Differ. 50, 291-294 (2011).

23. Ting-Toomey, S. in The Challenge of Facework: Cross-Cultural and Interpersonal Issues (ed. Ting-Toomey, S.) 1-14 (State Univ. New York Press, 1994).

24. Tan, C. B. Y., Stephen, I. D., Whitehead, R. \& Sheppard, E. You look familiar: how Malaysian Chinese recognize faces. PLoS ONE 7, e29714 (2012).

25. Chartier, C., McCarthy, R. \& Urry, H. The Psychological Science Accelerator (Association for Physical Science, 2018).

26. Chawla, D. S. A new 'accelerator' aims to bring big science to psychology. Science https://doi.org/10.1126/science.aar4464 (2017).

27. Moshontz, H. et al. The Psychological Science Accelerator: advancing psychology through a distributed collaborative network. Adv. Methods Pract. Psychol. Sci. 1, 501-515 (2018).

28. Widaman, K. F. On common factor and principal component representations of data: implications for theory and for confirmatory replications. Struct. Equ. Modeling 25, 829-847 (2018).

29. Hehman, E., Sutherland, C. A., Flake, J. K. \& Slepian, M. L.The unique contributions of perceiver and target characteristics in person perception. J. Pers. Soc. Psychol. 113, 513-529 (2017).

30. Sutherland, C. A., Rhodes, G., Burton, N. S. \& Young, A. W.Do facial first impressions reflect a shared social reality? Br. J. Psychol. 111, 215-232 (2020).

31. Oh, D., Dotsch, R., Porter, J. \& Todorov, A.Gender biases in impressions from faces: empirical studies and computational models. J. Exp. Psychol. Gen. 149, 323-342 (2020).

32. Oh, D., Shafir, E. \& Todorov, A. Economic status cues from clothes affect perceived competence from faces. Nat. Hum. Behav. 4, 287-293 (2020).

33. Collova, J. R., Sutherland, C. A. \& Rhodes, G. Testing the functional basis of first impressions: dimensions for children's faces are not the same as for adults' faces. J. Pers. Soc. Psychol. 117, 900-924 (2019).

34. Stolier, R. M., Hehman, E., Keller, M. D., Walker, M. \& Freeman, J. B. The conceptual structure of face impressions. Proc. Natl Acad. Sci. USA 115 9210-9215 (2018).

35. Stolier, R. M., Hehman, E. \& Freeman, J. B. A dynamic structure of social trait space. Trends Cogn. Sci. 22, 197-200 (2018).

36. Ma, D. S., Correll, J. \& Wittenbrink, B. The Chicago Face Database: a free stimulus set of faces and norming data. Behav. Res. Methods 47, 1122-1135 (2015).

37. Bainbridge, W. A., Isola, P. \& Oliva, A. The intrinsic memorability of face photographs. J. Exp. Psychol. Gen. 142, 1323-1334 (2013).

38. Sutherland, C. A. et al. Social inferences from faces: ambient images generate a three-dimensional model. Cognition 127, 105-118 (2013).

39. Burt, C. The factorial study of temperament traits. Br. J. Psychol. Stat. Sect. 1, 178-203 (1948).

40. Tucker, L. R. A Method for Synthesis of Factor Analysis Studies Personnel Research Section Report No. 984 (Department of the Army, 1951).

41. Davenport, E. C. Jr Significance testing of congruence coefficients: a good idea? Educ. Psychol. Meas. 50, 289-296 (1990).

42. Lorenzo-Seva, U. \& ten Berge, J. M. F. Tucker's congruence coefficient as a meaningful index of factor similarity. Methodology 2, 57-64 (2006).

43. Fabrigar, L. R., Wegener, D. T., MacCallum, R. C. \& Strahan, E. J. Evaluating the use of exploratory factor analysis in psychological research. Psychol. Methods 4, 272-299 (1999).

44. Park, H. S., Dailey, R. \& Lemus, D. The use of exploratory factor analysis and principal components analysis in communication research. Hum. Commun. Res. 28, 562-577 (2002).

45. Cliff, $\mathrm{N}$. The eigenvalues-greater-than-one rule and the reliability of components. Psychol. Bull. 103, 276-279 (1988).

46. Zwick, W. R. \& Velicer, W. F. Comparison of five rules for determining the number of components to retain. Psychol. Bull. 99, 432-442 (1986).

47. O'Connor, B. P. SPSS and SAS programs for determining the number of components using parallel analysis and Velicer's MAP test. Behav. Res. Methods Instrum. Comput. 32, 396-402 (2000).

48. Schmitt, T. A. Current methodological considerations in exploratory and confirmatory factor analysis. J. Psychoeduc. Assess. 29, 304-321 (2011)
49. Courtney, M. G. R. Determining the number of factors to retain in EFA: using the SPSS R-Menu v2.0 to make more judicious estimations. Pract. Assess. Res. Eval. 18, 1-14 (2013).

\section{Acknowledgements}

C.L. was supported by the Vienna Science and Technology Fund (WWTF VRG13-007); L.M.D. was supported by ERC 647910 (KINSHIP); D.I.B. and N.I. received funding from CONICET, Argentina; L.K., F.K. and Á. Putz were supported by the European Social Fund (EFOP-3.6.1.-16-2016-00004; 'Comprehensive Development for Implementing Smart Specialization Strategies at the University of Pécs'). K.U. and E. Vergauwe were supported by a grant from the Swiss National Science Foundation (PZ00P1_154911 to E. Vergauwe). T.G. is supported by the Social Sciences and Humanities Research Council of Canada (SSHRC). M.A.V. was supported by grants 2016-T1/SOC-1395 (Comunidad de Madrid) and PSI2017-85159-P (AEI/FEDER UE). K.B. was supported by a grant from the National Science Centre, Poland (number 2015/19/D/HS6/00641). J. Bonick and J.W.L. were supported by the Joep Lange Institute. G.B. was supported by the Slovak Research and Development Agency (APVV-17-0418). H.I.J. and E.S. were supported by a French National Research Agency 'Investissements d'Avenir' programme grant (ANR-15-IDEX-02). T.D.G. was supported by an Australian Government Research Training Program Scholarship. The Raipur Group is thankful to: (1) the University Grants Commission, New Delhi, India for the research grants received through its SAP-DRS (Phase-III) scheme sanctioned to the School of Studies in Life Science; and (2) the Center for Translational Chronobiology at the School of Studies in Life Science, PRSU, Raipur, India for providing logistical support. K. Ask was supported by a small grant from the Department of Psychology, University of Gothenburg. Y.Q. was supported by grants from the Beijing Natural Science Foundation (5184035) and CAS Key Laboratory of Behavioral Science, Institute of Psychology. N.A.C. was supported by the National Science Foundation Graduate Research Fellowship (R010138018). We acknowledge the following research assistants: J. Muriithi and J. Ngugi (United States International University Africa); E. Adamo, D. Cafaro, V. Ciambrone, F. Dolce and E. Tolomeo (Magna Græcia University of Catanzaro); E. De Stefano (University of Padova); S. A. Escobar Abadia (University of Lincoln); L. E. Grimstad (Norwegian School of Economics (NHH)); L. C. Zamora (Franklin and Marshall College); R. E. Liang and R. C. Lo (Universiti Tunku Abdul Rahman); A. Short and L. Allen (Massey University, New Zealand), A. Ateş, E. Güneş and S. Can Özdemir (Boğaziçi University); I. Pedersen and T. Roos (Åbo Akademi University); N. Paetz (Escuela de Comunicación Mónica Herrera); J. Green (University of Gothenburg); M. Krainz (University of Vienna, Austria); and B. Todorova (University of Vienna, Austria). The funders had no role in study design, data collection and analysis, decision to publish or preparation of the manuscript.

\section{Author contributions}

Conceptualization: B.C.J., L.M.D., J.K.F., J.P.W., J.B.F., S.Á.-S., H.I., S.M.J.J., H.L.

Data curation: B.C.J., L.M.D., N.C.A., N.G.B., Y.Q., J.W.L., K.G., G.M.M., J.G.L., J.B.F., P.C., A.P., N.P., S.P., M.M.S., B.P., M.J.B., V.K., J.P., D.S., S.C.W., J.V.V., P.S.F., C.R.C., N.A.C

Formal analysis: B.C.J., L.M.D., J.K.F., Y.Q., J.B.F.

Funding acquisition: N.C.O., Y.Q., J.W.L., C.C., J. Leongómez, O.R.S., E. Valderrama, M.V.-A., J.G.L., M.C.P., J.B.F., J.K.O., G.K., H.I., H.D.F., T.J.S.L., E. Vergauwe, K. Ask, K.W.T., M.I., C.L., P.S.F., C.R.C.

Investigation: B.C.J., L.M.D., M.T.L., J.A., I.L.G.N., N.G.B., S.C.L., F.F., M.L.W., C.P.C., M.A.V., S.A.S., N.C.O., D.P.C., A.W., Y.Q., H.M., P. Suavansri, T.R.E., J. Bonick, J.W.L., C.C., A. Kapucu, A. Karaaslan, J. Leongómez, O.R.S., E. Valderrama, M.V.-A., B.A., P. Szecsi, M. Andreychik, E.D.M., C.B., C.-P.H., Q.-L.L., L.A.V., K.B., K.G., I.S., S.S., R.A., C.M., W.V., Z.J., Q.W., G.M.M., I.D.S., J.G.L., M.C.P., J.D.A., E.H., S.Y.X., W.J.C., M. Seehuus, J.P.W., E.K., M.P.-P., A.E.B.-S., A.d.-G., I.G.-S., H.-H.W., J.B.F., D.W.O., V.S., T.E.S., C.A.L., C.L.C., A.K.P., J. Bavolar, P. Kačmár, I. Zakharov, S.Á.-S., E.B., M.T., K.S., C.D.C., J.W.S., J.K.O., A.-S.L., T.D.G., J.A.O., B.J.W.D., L.M.S., G.R., M.J.B., B.J., D.R., G.K., V.A.F., H.L.U., S.-C.C., G.P., Z.V., D.M.B.-B., H.I., N.V.d.L., C.B.Y.T., V.K., M.F.C., H.D.F., D.I.B., G.G., J.P., C.S., K.A.Ś., E.M.O.K., D.S., B.S., M. Sirota, G.V.S., T.J.S.L., K.U., E. Vergauwe, J.S., K. Ask, C.J.J.v.Z., A. Körner, S.C.W., J. Boudesseul, F.R.-D., K.L.R., N.M.M., K.R.B., D.W., A.R.G.-F., M. Anne, S.M.J.J., K.M.L., T.K.N., C.K.T., J.H.Z., A.D.R., L.K., M. Vianello, N.I., A.C., S.L., J. Lutz, M. Adamkovic, P.B., G.B., I.R., V.C., K.P., N.K.S., K.W.T., C.A.T., A.M.F., R.M.C.S.H., J.V.V., N.S.C.-F., M.F.-A., J.H., A.M., M. Sharifian, B.F., H.L., M.I., C.L., E.P., M. Voracek, J.O., E.M.G., A.A., A.A.Ö., M.T.C. B.B.-D., M.A.K., C.O., T.G., J.K.M., Y.D., X.Y., S. Alper, P.S.F., C.R.C., N.A.C.

Methodology: B.C.J., L.M.D., J.K.F., S.C.L., L.A.V., M. Seehuus, S. Azouaghe, A.B., J.E., J.P.W., J.B.F., C.A.L., C.D.C., K.H., B.J., J.W., G.K., H.I., T.B., N.V.d.L., H.D.F., J.P. F.M.A.W., S.M.J.J., H.L.

Project administration: B.C.J., L.M.D., N.G.B., S.C.L., M.L.W., M.G., A.S., N.C.O., A.W., Y.Q., H.M., R.M.S., J. Bonick, J.W.L., C.C., A. Kapucu, A. Karaaslan, J. Leongómez, O.R.S., E. Valderrama, M.V.-A., B.A., C.B., C.-P.H., L.A.V., K.B., K.G., I.S., S.S., I.D.S., M.C.P., S.Y.X., W.J.C., M. Seehuus, A.d.-G., I.G.-S., C.-C.K., J.B.F., D.W.O., C.A.L., J. Bavolar, P. Kačmár, I. Zakharov, K.S., C.D.C., J.W.S., J.L.B., J.A.O., B.J.W.D., M.J.B., B.J., D.R., G.P., Z.V., E.S., N.V.d.L., V.K., M.F.C., H.D.F., J.P., C.S., K.A.S., E.M.O.K., B.S., M. Sirota, T.J.S.L., K.U., E. Vergauwe, K. Ask, C.J.J.v.Z., S.C.W., J. Boudesseul, F.R.-D., K.L.R., D.W., S.M.J.J., C.K.T., J.H.Z., L.K., S.L., V.C., N.K.S., K.W.T., R.M.C.S.H., J.V.V., A.M., M. Sharifian, B.F., H.L., C.L., E.P., M. Voracek, A.A., A.A.Ö., M.A.K., T.G., X.Y., S. Alper, P.S.F., C.R.C., N.A.C. 
Resources: B.C.J., L.M.D., M.T.L., S.C.L., C.P.C., M.A.V., S.A.S., A.W., Y.Q., K. Ariyabuddhiphongs, S.J., H.M., P. Suavansri, N.T., R.M.S., C.C., A. Kapucu, J. Leongómez, M.V.-A., N.H., C.B., L.A.V., K.B., K.G., Z.J., G.M.M., I.D.S., J.G.L., S.Y.X., W.J.C., M. Seehuus, S. Azouaghe, A.B., J.E., A.d.-G., C.-C.K., J.B.F., C.A.L., A.K.P., P. Kačmár, I. Zakharov, E.B., K.S., C.D.C., J.K.O., J.L.B., B.J.W.D., D.R., W.W.A.S., S.-C.C., G.P., D.M.B.-B., T.B., C.B.Y.T., V.K., H.D.F., G.G., C.S., K.A.S., E.M.O.K., B.S., M. Sirota G.V.S., T.J.S.L., K.U., E. Vergauwe, K.J., K. Ask, J. Boudesseul, F.R.-D., N.M.M., S.M.J.J., C.K.T., A.D.R., F.K., Á.P., P.T., M. Vianello, A.C., S.L., J. Lutz, M. Adamkovic, P.B., V.C., A.M.F., R.M.C.S.H., J.V.V., N.S.C.-F., M.F.-A., A.M., M. Sharifian, H.L., C.L., M. Voracek, E.M.G., A.A.Ö., M.A.K., C.O., X.Y., S. Alper, P.S.F., C.R.C. Software: B.C.J., L.M.D., J.K.F., G.M.M., I.D.S., N.P., B.P., C.D.C., H.D.F., C.S., K.R.B., R.M.C.S.H., C.R.C., N.A.C Supervision: B.C.J., L.M.D., J.K.F., M.T.L., S.C.L., M.L.W., N.C.O., A.W., H.M., J.W.L., C.C., A. Kapucu, J. Leongómez, O.R.S., E. Valderrama, M.V.-A., M. Andreychik, E.D.M., C.B., L.A.V., K.B., I.D.S., M.C.P., E.H., W.J.C., M. Seehuus, C.-C.K., J.B.F., C.A.L., P. Kačmár, I. Zakharov, K.S., C.D.C., J.W.S., J.K.O., A.-S.L., J.L.B., J.A.O., B.J.W.D., M.J.B., H.I., V.K., M.F.C., H.D.F., J.P., C.S., E.M.O.K., D.S., B.S., M. Sirota, T.J.S.L., K.U., E. Vergauwe, K. Ask, C.J.J.v.Z., D.W., S.M.J.J., A.C., S.L., K.P., N.K.S., K.W.T., A.M.F., J.V.V., M. Sharifian, M.I., C.L., M. Voracek, A.A., A.A.Ö., M.A.K., S. Alper, P.S.F., C.R.C., N.A.C.

Validation: B.C.J., L.M.D., J.K.F., C.C., Q.W., S.Y.X., M. Seehuus, C.L.C., A.K.P., I. Zakharov, J.W.S., E.S., V.K., H.D.F., J.P., M. Sirota, E. Vergauwe, C.J.J.v.Z., P.T., J.H., M. Voracek, M.A.K.

Visualization: B.C.J., L.M.D., J.K.F., H.D.F., M.A.K., P.S.F. Writing (original draft): B.C.J., L.M.D., J.K.F., F.F., Y.Q., C.B., I.G.-S., J.B.F., K.S., B.J.W.D., G.K., H.L.U., H.I., H.D.F., D.I.B., J.P., C.S., D.S., K.L.R., S.M.J.J., A.D.R., N.K.S., J.O., A.A.Ö., M.A.K., P.S.F., N.A.C. Writing (review \& editing): B.C.J., L.M.D., J.K.F., M.T.L., J.A., I.L.G.N., S.C.L., F.F., M.L.W., M.A.V., A.S., D.P.C., A.W., Y.Q., K. Ariyabuddhiphongs, H.M., T.R.E., J. Bonick, J.W.L., C.C., J. Leongómez, B.A., N.H., P. Szecsi, M. Andreychik, E.D.M., C.B.,
N.L., L.A.V., K.B., I.S., S.S., Z.J., I.D.S., M.C.P., J.D.A., E.H., S.Y.X., W.J.C., M. Seehuus, S. Azouaghe, A.B., J.E., J.P.W., E.K., M.P.-P., A.E.B.-S., A.d.-G., J.B.F., V.S., T.E.S., C.A.L., C.L.C., P.C., P. Kujur, A.P., N.P., A.K.P., S.P., M.M.S., B.P., P. Kačmár, I. Zakharov, S.Á.-S., E.B., M.T., K.S., C.D.C., J.W.S., J.K.O., A.-S.L., J.L.B., T.D.G., J.A.O., B.J.W.D., G.R., M.J.B. K.H., B.J., G.K., V.A.F., H.L.U., G.P., Z.V., H.I., T.B., N.V.d.L., C.B.Y.T., V.K., M.F.C., H.D.F., D.I.B., G.G., C.S., E.M.O.K., D.S., B.S., M. Sirota, T.J.S.L., K.U., E. Vergauwe, J.S., K. Ask, C.J.J.v.Z., A. Körner, K.L.R., K.R.B., D.W., A.R.G.-F., S.M.J.J., T.K.N., C.K.T., J.H.Z., M. Vianello, N.I., M. Adamkovic, G.B., I.R., V.C., K.P., N.K.S., K.W.T., C.A.T., A.M.F., R.M.C.S.H., J.V.V., B.F., H.L., C.L., E.P., M. Voracek, J.O., E.M.G., A.A., A.A.Ö., B.B.-D., M.A.K., T.G., J.K.M., Y.D., P.S.F., C.R.C., N.A.C.

The following people did not indicate specific contributions: A.F.D., A.C.H., A.D.L.R.-G., D.R.F., D.T., E.T., E.G.-S., H.I.J., I. Zettler, I.R.P., J.A.M.-R., J.D.L., L.N., L.F.A., M.A.C.V., M.M.A., M.L.B.-G., M.H.S., N.O.R., P.P., P.F., R.J.M., S.G., S.J.C., T.H., V.K.M.S., W.-J.Y.

Competing interests

The authors declare no competing interests.

Additional information

Supplementary information is available for this paper at https://doi.org/10.1038/ s41562-020-01007-2.

Correspondence and requests for materials should be addressed to B.C.J.

Peer review information Primary Handling Editor: Stavroula Kousta.

Reprints and permissions information is available at www.nature.com/reprints.

๑) The Author(s), under exclusive licence to Springer Nature Limited 2021

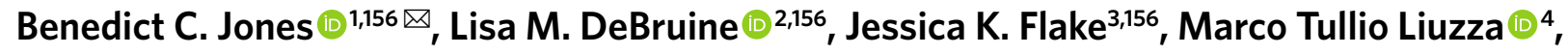
Jan Antfolk $\oplus^{5}$, Nwadiogo C. Arinze $\mathbb{1}^{6}$, Izuchukwu L. G. Ndukaihe ${ }^{6}$, Nicholas G. Bloxsom? Savannah C. Lewis $\oplus^{7}$, Francesco Foroni ${ }^{8}{ }^{8}$, Megan L. Willis ${ }^{8}{ }^{8}$, Carmelo P. Cubillas ${ }^{9}$, Miguel A. Vadillo ${ }^{\circ}{ }^{9}$, Enrique Turiegano ${ }^{10}{ }^{10}$, Michael Gilead"1 Almog Simchon ${ }^{11}$, S. Adil Saribay $\oplus^{12}$, Nicholas C. Owsley ${ }^{13}$, Chaning Jang ${ }^{13}$, Georgina Mburu ${ }^{13}$, Dustin P. Calvillo ${ }^{14}$, Anna Wlodarczyk $\oplus^{15}$, Yue $\mathrm{Qi}^{16}$, Kris Ariyabuddhiphongs ${ }^{17}{ }^{17}$, Somboon Jarukasemthawe ${ }^{17}$, Harry Manley ${ }^{17}$, Panita Suavansri ${ }^{17}$, Nattasuda Taephant ${ }^{17}$, Ryan M. Stolier ${ }^{18}$, Thomas R. Evans ${ }^{19}{ }^{17}$, Judson Bonick $\oplus^{20}$, Jan W. Lindemans ${ }^{20}$, Logan F. Ashworth ${ }^{21}$, Amanda C. Hahn ${ }^{21}$, Coralie Chevallier $\circledast^{22}$, Aycan Kapucu ${ }^{23}$, Aslan Karaaslan ${ }^{23}$, Juan David Leongómez ${ }^{24}$, Oscar R. Sánchez ${ }^{24}$, Eugenio Valderrama ${ }^{24}$, Milena Vásquez-Amézquita ${ }^{24}$, Nandor Hajdu $(25,26$, Balazs Aczel ${ }^{26}$, Peter Szecsi $\odot{ }^{26}$, Michael Andreychik $\left({ }^{27}\right.$, Erica D. Musser $\left({ }^{28}\right.$, Carlota Batres $\left({ }^{29}\right.$, Chuan-Peng Hu $\oplus^{30}$, Qing-Lan Liu ${ }^{31}$, Nicole Legate $\odot^{32}$, Leigh Ann Vaughn $\oplus^{33}$, Krystian Barzykowski $\odot^{34}$, Karolina Golik ${ }^{34}$, Irina Schmid $\oplus^{35}$, Stefan Stieger $\left({ }^{35}\right.$, Richard Artner $\left(\oplus^{36}\right.$, Chiel Mues $\oplus^{36}$, Wolf Vanpaemel ${ }^{37}$, Zhongqing Jiang $\oplus^{38}$, Qi Wu ${ }^{38}$, Gabriela M. Marcu $\oplus^{39}$, Ian D. Stephen $\mathbb{1}^{40}$, Jackson G. Lu $\oplus^{41}$, Michael C. Philipp ${ }^{42}$, Jack D. Arnal ${ }^{43}$, Eric Hehman ${ }^{3}$, Sally Y. Xie ${ }^{3}$, William J. Chopik ${ }^{44}$, Martin Seehuus ${ }^{45}$, Soufian Azouaghe ${ }^{46,47}$, Abdelkarim Belhaj ${ }^{46}$, Jamal Elouafa ${ }^{46}$, John P. Wilson ${ }^{48}{ }^{48}$, Elliott Kruse ${ }^{49}$, Marietta Papadatou-Pastou ${ }^{50}{ }^{50}$, Anabel De La Rosa-Gómez ${ }^{51}$, Alan E. Barba-Sánchez ${ }^{51}$, Isaac González-Santoyo ${ }^{552}$, Tsuyueh Hsu $\oplus^{53}$, Chun-Chia Kung ${ }^{53}$, Hsiao-Hsin Wang ${ }^{53}$, Jonathan B. Freeman ${ }^{54}$, Dong Won Oh ${ }^{55}$, Vidar Schei ${ }^{56}$, Therese E. Sverdrup ${ }^{(156}$, Carmel A. Levitan ${ }^{57}{ }^{57}$, Corey L. Cook ${ }^{58}$, Priyanka Chandel $\oplus^{59}$, Pratibha Kujur $\oplus^{59}$, Arti Parganiha $\oplus^{59}$, Noorshama Parveen $\oplus^{59}$, Atanu Kumar Pati $\oplus^{59}$, Sraddha Pradhan ${ }^{59}$, Margaret M. Singh ${ }^{59}$, Babita Pande ${ }^{60}$, Jozef Bavolar ${ }^{61}$, Pavol Kačmár $\oplus^{61}$, Ilya Zakharov ${ }^{62}$, Sara Álvarez-Solas ${ }^{63}$, Ernest Baskin ${ }^{64}$, Martin Thirkettle ${ }^{65}$, Kathleen Schmidt ${ }^{66}$, Cody D. Christopherson ${ }^{67}{ }^{67}$, Trinity Leonis ${ }^{67}$, Jordan W. Suchow ${ }^{68}$, Jonas K. Olofsson $\circledast^{69}$, Teodor Jernsäther ${ }^{69}{ }^{69}$, Ai-Suan Lee ${ }^{70}$, Jennifer L. Beaudry ${ }^{71}$, Taylor D. Gogan ${ }^{71}$, Julian A. Oldmeadow ${ }^{71}$, Benjamin Balas ${ }^{72}$, Laura M. Stevens ${ }^{73}$, 
Melissa F. Colloff ${ }^{73}$, Heather D. Flowe ${ }^{73}$, Sami Gülgöz $\mathbb{C}^{74}$, Mark J. Brandt ${ }^{75}$, Karlijn Hoyer ${ }^{75}$, Bastian Jaeger ${ }^{75}$, Dongning Ren ${ }^{75}$, Willem W. A. Sleegers ${ }^{75}$, Joeri Wissink ${ }^{75}$,

Gwenaël Kaminski ${ }^{76}$, Victoria A. Floerke ${ }^{77}$, Heather L. Urry ${ }^{77}$, Sau-Chin Chen ${ }^{78}$, Gerit Pfuhl ${ }^{79}$, Zahir Vally ${ }^{80}$, Dana M. Basnight-Brown ${ }^{81}$, Hans I. Jzerman ${ }^{47}$, Elisa Sarda ${ }^{47}$, Lison Neyroud ${ }^{82}$,

Touhami Badidi ${ }^{83}$, Nicolas Van der Linden ${ }^{84}$, Chrystalle B. Y. Tan ${ }^{85}{ }^{85}$, Vanja Kovic ${ }^{86}$, Waldir Sampaio ${ }^{87}$, Paulo Ferreira ${ }^{88}$, Diana Santos ${ }^{88}$, Debora I. Burin ${ }^{89}{ }^{89}$, Gwendolyn Gardiner ${ }^{90}$, John Protzko ${ }^{91}$, Christoph Schild ${ }^{92}$, Karolina A. Ścigała92, Ingo Zettler (10 ${ }^{92}$, Erin M. O'Mara Kunz ${ }^{10}{ }^{93}$, Daniel Storage ${ }^{94}$, Fieke M. A. Wagemans ${ }^{95}$, Blair Saunders ${ }^{96}$, Miroslav Sirota ${ }^{97}$, Guyan V. Sloane ${ }^{97}$, Tiago J. S. Lima ${ }^{98}$, Kim Uittenhove ${ }^{99}$, Evie Vergauwe ${ }^{99}$, Katarzyna Jaworska ${ }^{(1)}$, Julia Stern ${ }^{100}$, Karl Ask ${ }^{101}$, Casper J. J. van Zyl' ${ }^{102}$, Anita Körner ${ }^{103}$, Sophia C. Weissgerber ${ }^{103}$, Jordane Boudesseul (104 , Fernando Ruiz-Dodobara104, Kay L. Ritchie ${ }^{105}$, Nicholas M. Michalak (iD) ${ }^{106}$, Khandis R. Blake ${ }^{107,108}$, David White ${ }^{107}$, Alasdair R. Gordon-Finlayson ${ }^{109}$, Michele Anne ${ }^{110}$, Steve M. J. Janssen ${ }^{110}$, Kean Mun Lee ${ }^{110}$, Tonje K. Nielsen ${ }^{111}$, Christian K. Tamnes ${ }^{111}$, Janis H. Zickfeld (112, Anna Dalla Rosa ${ }^{113}$, Michelangelo Vianello ${ }^{113}$, Ferenc Kocsor ${ }^{114}$, Luca Kozma ${ }^{114}$, Ádám Putz ${ }^{114}$, Patrizio Tressoldi ${ }^{115}$, Natalia Irrazabal ${ }^{116}$, Armand Chatard ${ }^{117}$, Samuel Lins ${ }^{118}$, Isabel R. Pinto ${ }^{118}$, Johannes Lutz ${ }^{119}$, Matus Adamkovic (1) 120, Peter Babincak (120,

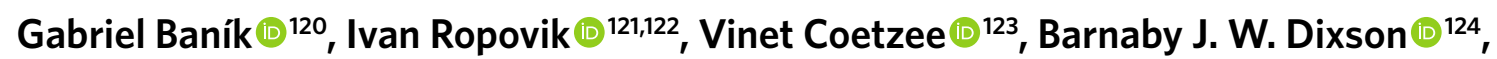
Gianni Ribeiro ${ }^{124}$, Kim Peters ${ }^{124}$, Niklas K. Steffens ${ }^{124}$, Kok Wei Tan ${ }^{125}$, Christopher A. Thorstenson ${ }^{126}$, Ana Maria Fernandez ${ }^{127}$, Rafael M. C. S. Hsu ${ }^{128}$, Jaroslava V. Valentova ${ }^{128}$, Marco A. C. Varella (id) ${ }^{128}$, Nadia S. Corral-Frías (1) ${ }^{129}$, Martha Frías-Armenta ${ }^{129}$, Javad Hatami ${ }^{130}$, Arash Monajem ${ }^{130}$, MohammadHasan Sharifian ${ }^{130}$, Brooke Frohlich ${ }^{131}$, Hause Lin ${ }^{132}$, Michael Inzlicht ${ }^{132}$, Ravin Alaei ${ }^{132}$, Nicholas O. Rule ${ }^{132}$, Claus Lamm ${ }^{133}$, Ekaterina Pronizius ${ }^{133}$, Martin Voracek ${ }^{133}$, Jerome Olsen ${ }^{134}$, Erik Mac Giolla ${ }^{135}$, Aysegul Akgoz ${ }^{136}$, Asil A. Özdoğru ${ }^{136}$, Matthew T. Crawford ${ }^{137}$, Brooke Bennett-Day ${ }^{138}$, Monica A. Koehn ${ }^{13}{ }^{139}$, Ceylan Okan ${ }^{140}$, Tripat Gill ${ }^{141}$, Jeremy K. Miller (1) ${ }^{142}$, Yarrow Dunham ${ }^{143}$, Xin Yang ${ }^{143}$, Sinan Alper ${ }^{144}$, Martha Lucia Borras-Guevara ${ }^{145}$, Sun Jun Cai ${ }^{146}$, Dong Tiantian (1) ${ }^{146}$, Alexander F. Danvers ${ }^{147}$, David R. Feinberg ${ }^{148}$, Marie M. Armstrong ${ }^{148}$, Eva Gilboa-Schechtman ${ }^{149}$, Randy J. McCarthy ${ }^{150}$, Jose Antonio Muñoz-Reyes ${ }^{151}$, Pablo Polo ${ }^{151}$, Victor K. M. Shiramazu ${ }^{152}$, Wen-Jing Yan ${ }^{153}$, Lilian Carvalho ${ }^{154}$, Patrick S. Forscher ${ }^{82}$, Christopher R. Chartier ${ }^{7}{ }^{7}$ and Nicholas A. Coles ${ }^{155}$

\footnotetext{
'School of Psychological Sciences and Health, University of Strathclyde, Glasgow, UK. Institute of Neuroscience and Psychology, University of Glasgow, Glasgow, UK. ${ }^{3}$ Department of Psychology, McGill University, Montreal, Québec, Canada. ${ }^{4}$ Department of Medical and Surgical Sciences, Magna Græcia University of Catanzaro, Catanzaro, Italy. ${ }^{5}$ Faculty of Arts, Psychology and Theology, Åbo Akademi University, Turku, Finland. ${ }^{6}$ Department of Psychology, Alex Ekwueme Federal University Ndufu Alike, Ikwo, Nigeria. ${ }^{7}$ Department of Psychology, Ashland University, Danville, CA, USA. ${ }^{8}$ School of Behavioural and Health Sciences, Australian Catholic University, Sydney, New South Wales, Australia. ' Department of Basic Psychology, Autonomous University of Madrid, Madrid, Spain. ${ }^{10}$ Department of Biology, Autonomous University of Madrid, Madrid, Spain. "Department of Psychology, Ben-Gurion University of the Negev, Beersheba, Israel. ${ }^{12}$ Department of Psychology, Boğaziçi University, Beșiktaș, Turkey. ${ }^{13}$ Busara Center for Behavioral Economics, Nairobi, Kenya. ${ }^{14}$ Psychology Department, California State University San Marcos, San Marcos, CA, USA. ${ }^{15}$ School of Psychology, Catholic University of the North, Antofagasta, Chile. ${ }^{16}$ Department of Psychology, Renmin University of China, Beijing, China. ${ }^{17}$ Faculty of Psychology, Chulalongkorn University, Bangkok, Thailand. ${ }^{18}$ Department of Psychology, Columbia University, New York, NY, USA. ${ }^{19}$ School of Psychological, Social and Behavioural Sciences, Coventry University, Coventry, UK. ${ }^{20}$ Center for Advanced Hindsight, Duke University, Durham, NC, USA. ${ }^{21}$ Department of Psychology, Humboldt State University, Arcata, CA, USA. ${ }^{22}$ Laboratoire de Neurosciences Cognitives et Computationnelles, Département d'Études Cognitives, INSERM U960, École Normale Supérieure, Paris, France. ${ }^{23}$ Psychology Department, Ege University, İzmir, Turkey. ${ }^{24}$ Faculty of Psychology, Universidad El Bosque, Bogotá, Colombia. ${ }^{25}$ Doctoral School of Psychology, ELTE Eötvös Loránd University, Budapest, Hungary. ${ }^{26}$ Institute of Psychology, ELTE Eötvös Loránd University, Budapest, Hungary. ${ }^{27}$ Department of Psychology, Fairfield University, Fairfield, CT, USA. ${ }^{28}$ Department of Psychology, Florida International University, Miami, FL, USA. ${ }^{29}$ Department of Psychology, Franklin and Marshall College, Lancaster, PA, USA. ${ }^{30}$ Leibniz Institute for Resilience Research, Mainz, Germany. ${ }^{31}$ Department of Psychology, Hubei University, Wuhan, China. ${ }^{32}$ Department of Psychology, Illinois Institute of Technology, Chicago, IL, USA. ${ }^{33}$ Department of Psychology, Ithaca College, Ithaca, NY, USA. ${ }^{34}$ Institute of Psychology, Jagiellonian University, Kraków, Poland. ${ }^{35}$ Department of Psychology and Psychodynamics, Karl
} 
Landsteiner University of Health Sciences, Krems an der Donau, Austria. ${ }^{36}$ Research Group of Quantitative Psychology and Individual Differences, Katholieke Universiteit Leuven, Leuven, Belgium. ${ }^{37}$ Faculty of Psychology and Educational Sciences, Katholieke Universiteit Leuven, Leuven, Belgium. ${ }^{38}$ Department of Psychology, Liaoning Normal University, Dalian, China. ${ }^{39}$ Department of Psychology, Lucian Blaga University of Sibiu, Sibiu, Romania. ${ }^{40}$ Department of Psychology, Macquarie University, Sydney, New South Wales, Australia. ${ }^{41}$ Sloan School of Management, Massachusetts Institute of Technology, Cambridge, MA, USA. ${ }^{42}$ School of Psychology, Massey University, Palmerston North, New Zealand. ${ }^{43}$ Psychology Department, McDaniel College, Westminster, CO, USA. ${ }^{44}$ Department of Psychology, Michigan State University, East Lansing, MI, USA. ${ }^{45}$ Department of Psychology, Middlebury College, Middlebury, VT, USA. ${ }^{46}$ Department of Psychology, Mohammed V University in Rabat, Rabat, Morocco. ${ }^{47} \mathrm{LIP} / \mathrm{PC} 2 \mathrm{~S}$, Université Grenoble Alpes, Grenoble, France. ${ }^{48}$ Psychology Department, Montclair State University, Montclair, NJ, USA. ${ }^{49}$ EGADE Business School, Monterrey Institute of Technology and Higher Education, Monterrey, Mexico. ${ }^{50}$ School of Education, National and Kapodistrian University of Athens, Athens, Greece. ${ }^{51}$ School of Higher Studies Iztacala, National Autonomous University of Mexico, Mexico City, Mexico. ${ }^{52}$ Department of Psychology, National Autonomous University of Mexico, Mexico City, Mexico. ${ }^{53}$ Department of Psychology, National Cheng Kung University, Tainan City, Taiwan. ${ }^{54}$ Department of Psychology and Center for Neural Science, New York University, New York, NY, USA. ${ }^{55}$ Department of Psychology, New York University, New York, NY, USA. ${ }^{56}$ Department of Strategy and Management, Norwegian School of Economics (NHH), Bergen, Norway. ${ }^{57}$ Department of Cognitive Science, Occidental College, Los Angeles, CA, USA. ${ }^{58}$ Department of Psychology, Pacific Lutheran University, Tacoma, WA, USA. ${ }^{59}$ School of Studies in Life Science, Pandit Ravishankar Shukla University, Raipur, India. ${ }^{60} \mathrm{Center}$ for Basic Sciences, Pandit Ravishankar Shukla University, Raipur, India. ${ }^{61}$ Department of Psychology, Pavol Jozef Šafárik University in Košice, Košice, Slovakia. ${ }^{62}$ Developmental Behavioral Genetics Lab, Psychological Institute of Russian Academy of Education, Moscow, Russia. ${ }^{63}$ Facultad de Ciencias de la Vida, Universidad Regional Amazónica Ikiam, Guayaquil, Ecuador. ${ }^{64}$ Department of Food Marketing, Saint Joseph's University, Philadelphia, PA, USA. ${ }^{65}$ Centre for Behavioural Science and Applied Psychology, Sheffield Hallam University, Sheffield, UK. ${ }^{66}$ School of Psychological and Behavioral Sciences, Southern Illinois University, Carbondale, IL, USA. ${ }^{67}$ Psychology Department, Southern Oregon University, Ashland, OR, USA. ${ }^{68}$ School of Business, Stevens Institute of Technology, Hoboken, NJ, USA. ${ }^{69}$ Department of Psychology, Stockholm University, Stockholm, Sweden. ${ }^{70}$ Department of Psychology, Sunway University, Subang Jaya, Malaysia. ${ }^{71}$ Department of Psychological Sciences, Swinburne University of Technology, Melbourne, Victoria, Australia. ${ }^{72}$ Department of Psychology, North Dakota State University, Fargo, ND, USA. ${ }^{73}$ School of Psychology, University of Birmingham, Birmingham, UK. ${ }^{74}$ Koç University, Istanbul, Turkey. ${ }^{75}$ Department of Social Psychology, Tilburg University, Tilburg, the Netherlands. ${ }^{76} \mathrm{CLLE}$, Toulouse University, Toulouse, France. ${ }^{77}$ Department of Psychology, Tufts University, Medford, MA, USA. ${ }^{78}$ Department of Human Development and Psychology, Tzu-Chi University, Hualien, Taiwan. ${ }^{79}$ Department of Psychology, UiT The Arctic University of Norway, Tromsø, Norway. ${ }^{80}$ Department of Psychology and Counseling, United Arab Emirates University, Abu Dhabi, United Arab Emirates. ${ }^{81}$ United States International University Africa, Nairobi, Kenya. ${ }^{82}$ Department of Psychology, Université Grenoble Alpes, Saint-Martin-d'Hères, France. ${ }^{83}$ Department of Psychology, Université lbn Tofail, Kénitra, Morocco. ${ }^{84}$ Center for Social and Cultural Psychology, Université Libre de Bruxelles, Brussels, Belgium. ${ }^{85}$ Department of Community and Family Medicine, Universiti Malaysia Sabah, Kota Kinabalu, Malaysia. ${ }^{86}$ Department of Psychology, Faculty of Philosophy, University of Belgrade, Belgrade, Serbia. ${ }^{87}$ Universidade Federal de São Carlos, São Paulo, Brazil. ${ }^{88}$ Universidade Federal da Grande Dourados, Dourados, Brazil. ${ }^{89}$ nstituto de Investigaciones, Facultad de Psicologia, Universidad de Buenos Aires, Buenos Aires, Argentina. ${ }^{90}$ Department of Psychology, University of California, Riverside, Riverside, CA, USA. ${ }^{91}$ Department of Psychological and Brain Sciences, University of California, Santa Barbara, Santa Barbara, CA, USA. ${ }^{92}$ Department of Psychology, University of Copenhagen, Copenhagen, Denmark. ${ }^{93}$ Department of Psychology, University of Dayton, Dayton, OH, USA. ${ }^{94}$ Department of Psychology, University of Denver, Denver, CO, USA. ${ }^{95}$ Institute for Socio-Economics, University of Duisburg-Essen, Essen, Germany. ${ }^{96}$ School of Social Sciences, University of Dundee, Dundee, UK. ${ }^{97}$ Department of Psychology, University of Essex, Colchester, UK. ${ }^{98}$ Department of Social and Work Psychology, University of Brasília, Brasília, Brazil. ${ }^{99}$ Faculty of Psychology and Educational Sciences, University of Geneva, Geneva, Switzerland. ${ }^{100}$ Department of Psychology, University of Goettingen, Goettingen, Germany. ${ }^{101}$ Department of Psychology, University of Gothenburg, Gothenburg, Sweden. ${ }^{102}$ Department of Psychology, University of Johannesburg, Johannesburg, South Africa. ${ }^{103}$ Department of Psychology, University of Kassel, Kassel, Germany. ${ }^{104}$ Institute of Scientific Research, Faculty of Psychology, University of Lima, Lima, Peru. ${ }^{105}$ School of Psychology, University of Lincoln, Lincoln, UK. ${ }^{106}$ Department of Psychology, University of Michigan, Ann Arbor, MI, USA. ${ }^{107}$ Evolution and Ecology Research Centre, University of New South Wales Sydney, Sydney, New South Wales, Australia. ${ }^{108}$ Melbourne School of Psychological Sciences, University of Melbourne, Melbourne, Victoria, Australia. ${ }^{109}$ Faculty of Health, Education and Society, University of Northampton, Northampton, UK. ${ }^{10}$ School of Psychology, University of Nottingham Malaysia, Semenyih, Malaysia. ${ }^{11}$ Department of Psychology, University of Oslo, Oslo, Norway. ${ }^{12}$ Department of Management, Aarhus University, Aarhus, Denmark. ${ }^{113}$ Department of Philosophy, Sociology, Education and Applied Psychology, University of Padova, Padova, Italy. ${ }^{114}$ Institute of Psychology, University of Pécs, Pécs, Hungary. ${ }^{115}$ Department of General Psychology, University of Padova, Padova, Italy. ${ }^{116}$ Faculty of Social Sciences, University of Palermo, Buenos Aires, Argentina. ${ }^{17}$ Psychology Department, University of Poitiers, Poitiers, France. ${ }^{118}$ Department of Psychology, University of Porto, Porto, Portugal. ${ }^{119}$ Department of Psychology, University of Potsdam, Potsdam, Germany. ${ }^{120}$ Institute of Psychology, Faculty of Arts, University of Presov, Presov, Slovakia. ${ }^{121}$ Faculty of Education, University of Presov, Presov, Slovakia. ${ }^{122}$ Institute for Research and Development of Education, Faculty of Education, Charles University, Prague, Czechia. ${ }^{123}$ Department of Biochemistry, Genetics and Microbiology, University of Pretoria, Pretoria, South Africa. ${ }^{124}$ School of Psychology, University of Queensland, Brisbane, Queensland, Australia. ${ }^{125}$ School of Psychology and Clinical Language Sciences, University of Reading Malaysia, Johor, Malaysia. ${ }^{126}$ Department of Clinical and Social Sciences in Psychology, University of Rochester, Rochester, NY, USA. ${ }^{127}$ School of Psychology, University of Santiago, Chile, Santiago, Chile. ${ }^{128}$ Institute of Psychology, Department of Experimental Psychology, University of São Paulo, São Paulo, Brazil. ${ }^{129}$ Department of Psychology, University of Sonora, Hermosillo, Mexico. ${ }^{130}$ Department of Psychology, University of Tehran, Tehran, Iran. ${ }^{131}$ Department of Psychology, University of Tennessee, Knoxville, Knoxville, TN, USA. ${ }^{132}$ Department of Psychology, University of Toronto, Toronto, Ontario, Canada. ${ }^{133}$ Department of Cognition, Emotion, and Methods in Psychology, Faculty of Psychology, University of Vienna, Vienna, Austria. ${ }^{134}$ Department of Applied Psychology: Work, Education and Economy, Faculty of Psychology, University of Vienna, Vienna, Austria. ${ }^{135}$ Department of Behavioral Sciences, University West, Trollhättan, Sweden. ${ }^{136}$ Department of Psychology, Üsküdar University, Istanbul, Turkey. ${ }^{137}$ School of Psychology, Victoria University of Wellington, Wellington, New Zealand. ${ }^{138}$ Department of Psychology, Wesleyan College, Middletown, CT, USA. ${ }^{139}$ Discipline of Psychology, Faculty of Health, University of Canberra, Canberra, Australian Capital Territory, Australia. ${ }^{140}$ School of Social Science and Psychology, Western Sydney University, Sydney, New South Wales, Australia. ${ }^{141}$ Lazaridis School of Business and Economics, Wilfrid Laurier University, Waterloo, Ontario, Canada. ${ }^{142}$ Department of Psychology, Willamette University, Salem, OR, USA. ${ }^{143}$ Department of Psychology, Yale University, New Haven, CT, USA. ${ }^{144}$ Department of Psychology, Yasar University, Izmir, Turkey. ${ }^{145}$ University of St. Andrews, St. Andrews, UK. ${ }^{146}$ Qufu Normal University, Jining, China. ${ }^{147}$ University of Oklahoma, Norman, OK, USA. ${ }^{148}$ Department of Psychology, Neuroscience, and Behaviour, McMaster University, Hamilton, Ontario, Canada. ${ }^{149}$ Bar-Illan University, Tel Aviv, Israel. ${ }^{150}$ Northern Illinois University, DeKalb, IL, USA. ${ }^{151}$ Playa Ancha University of Educational Sciences, Valparaiso, Chile. ${ }^{152}$ Federal University of Rio Grande do Norte, Rio Grande do Norte, Brazil. ${ }^{153}$ Wenzhou University, Wenzhou, China. ${ }^{154} \mathrm{FGV} / \mathrm{EAESP}$, Sao Paulo, Brazil. ${ }^{155} \mathrm{H}$ arvard Kennedy School, Harvard University, Cambridge, MA, USA. ${ }^{156}$ These authors contributed equally: Benedict C. Jones, Lisa M. DeBruine, Jessica K. Flake. ${ }^{凶}$ e-mail: psysciacc.001@gmail.com 


\section{Reporting Summary}

Nature Research wishes to improve the reproducibility of the work that we publish. This form provides structure for consistency and transparency in reporting. For further information on Nature Research policies, see our Editorial Policies and the Editorial Policy Checklist.

\section{Statistics}

For all statistical analyses, confirm that the following items are present in the figure legend, table legend, main text, or Methods section.

n/a Confirmed

$\square$ The exact sample size $(n)$ for each experimental group/condition, given as a discrete number and unit of measurement

$\square$ A statement on whether measurements were taken from distinct samples or whether the same sample was measured repeatedly

$\square$ The statistical test(s) used AND whether they are one- or two-sided

$\square$ Only common tests should be described solely by name; describe more complex techniques in the Methods section.

$\square$ A description of all covariates tested

$\square$ \A description of any assumptions or corrections, such as tests of normality and adjustment for multiple comparisons

$\square$ A full description of the statistical parameters including central tendency (e.g. means) or other basic estimates (e.g. regression coefficient)

$\triangle$ AND variation (e.g. standard deviation) or associated estimates of uncertainty (e.g. confidence intervals)

Х For null hypothesis testing, the test statistic (e.g. $F, t, r$ ) with confidence intervals, effect sizes, degrees of freedom and $P$ value noted

Wive $P$ values as exact values whenever suitable.

\ $\square$ For Bayesian analysis, information on the choice of priors and Markov chain Monte Carlo settings

$\square$ \ For hierarchical and complex designs, identification of the appropriate level for tests and full reporting of outcomes

$\square \bigotimes$ Estimates of effect sizes (e.g. Cohen's $d$, Pearson's $r$ ), indicating how they were calculated

Our web collection on statistics for biologists contains articles on many of the points above.

\section{Software and code}

Policy information about availability of computer code

Data collection DeBruine. L.M. (2019, April 9). Experimentum: Beta release 1 (Version v.0.1). Zenodo. http://doi.org/10.5281/zenodo.2634356

Data analysis https://osf.io/87rbg/

For manuscripts utilizing custom algorithms or software that are central to the research but not yet described in published literature, software must be made available to editors and reviewers. We strongly encourage code deposition in a community repository (e.g. GitHub). See the Nature Research guidelines for submitting code \& software for further information.

\section{Data}

Policy information about availability of data

All manuscripts must include a data availability statement. This statement should provide the following information, where applicable:

- Accession codes, unique identifiers, or web links for publicly available datasets

- A list of figures that have associated raw data

- A description of any restrictions on data availability 


\section{Field-specific reporting}

Please select the one below that is the best fit for your research. If you are not sure, read the appropriate sections before making your selection.
$\square$ Life sciences
Behavioural \& social sciences
Ecological, evolutionary \& environmental sciences

For a reference copy of the document with all sections, see nature.com/documents/nr-reporting-summary-flat.pdf

\section{Behavioural \& social sciences study design}

All studies must disclose on these points even when the disclosure is negative.

Study description

Research sample

Sampling strategy

Data collection

Timing

Data exclusions

Non-participation

Randomization
Quantitative analysis of ratings data

Student-type samples collected in eleven different world regions

Opportunistic sampling by individual research groups

Rating of faces on one of thirteen randomly determined traits

Throughout 2019

All data exclusions were described in the stage one protocol and in the analysis code

These data are given in the analysis code and output

Participants were randonly allocated to rate one triat

\section{Reporting for specific materials, systems and methods}

We require information from authors about some types of materials, experimental systems and methods used in many studies. Here, indicate whether each material, system or method listed is relevant to your study. If you are not sure if a list item applies to your research, read the appropriate section before selecting a response.

\begin{tabular}{l|l} 
Materials \& experimental system \\
\hline $\mathrm{n} / \mathrm{a}$ & Involved in the study \\
$\searrow$ & $\square$ Antibodies \\
$\square$ & $\square$ Eukaryotic cell lines \\
$\square$ & $\square$ Palaeontology and archaeology \\
$\square$ & $\square$ Animals and other organisms \\
$\square$ & $\square$ Human research participants \\
$\searrow$ & $\square$ Clinical data \\
$\square$ & $\square$ Dual use research of concern
\end{tabular}

\begin{tabular}{l|l} 
Methods \\
\hline n/a & Involved in the study \\
$\searrow$ & $\square$ ChIP-seq \\
$\bigotimes$ & $\square$ Flow cytometry \\
$\square$ & $\square$ MRI-based neuroimaging
\end{tabular}

\section{Human research participants}

Policy information about studies involving human research participants

Population characteristics

Recruitment

Ethics oversight
Raters from a range of geographic regions and countries. Region and country information are reported.

Opportunistic recruitment by individual labs.

main ethics approval was from University of Glasgow, although some individual groups also obtained their own thicas approvals.

Note that full information on the approval of the study protocol must also be provided in the manuscript. 